\title{
Accurate simulation of surfaces and interfaces of ten FCC metals and steel using Lennard-Jones potentials
}

\author{
Krishan Kanhaiya $\mathbb{D}^{1}$, Seonghan Kim $\mathbb{D}^{2}$, Wonpil Im $\mathbb{D}^{2}$ and Hendrik Heinz $\mathbb{D}^{1 凶}$
}

The earlier integration of validated Lennard-Jones (LJ) potentials for $8 \mathrm{fcc}$ metals into materials and biomolecular force fields has advanced multiple research fields, for example, metal-electrolyte interfaces, recognition of biomolecules, colloidal assembly of metal nanostructures, alloys, and catalysis. Here we introduce 12-6 and 9-6 LJ parameters for classical all-atom simulations of 10 further fcc metals (Ac, Ca (a), Ce ( $\gamma)$, Es $(\beta), \mathrm{Fe}(\gamma), \mathrm{Ir}, \mathrm{Rh}, \mathrm{Sr}(\mathrm{a}), \mathrm{Th}(\mathrm{a}), \mathrm{Yb}(\beta))$ and stainless steel. The parameters reproduce lattice constants, surface energies, water interfacial energies, and interactions with (bio)organic molecules in 0.1 to $5 \%$ agreement with experiment, as well as qualitative mechanical properties under standard conditions. Deviations are reduced up to a factor of one hundred in comparison to earlier Lennard-Jones parameters, embedded atom models, and density functional theory. We also explain a quantitative correlation between atomization energies from experiments and surface energies that supports parameter development. The models are computationally very efficient and applicable to an exponential space of alloys. Compatibility with a wide range of force fields such as the Interface force field (IFF), AMBER, CHARMM, COMPASS, CVFF, DREIDING, OPLS-AA, and PCFF enables reliable simulations of nanostructures up to millions of atoms and microsecond time scales. User-friendly model building and input generation are available in the CHARMM-GUI Nanomaterial Modeler. As a limitation, deviations in mechanical properties vary and are comparable to DFT methods. We discuss the incorporation of reactivity and features of the electronic structure to expand the range of applications and further increase the accuracy.

npj Computational Materials (2021)7:17; https://doi.org/10.1038/s41524-020-00478-1

\section{INTRODUCTION}

Metals and alloys have been historically used in jewelry, accessories, load-bearing structures, and electrical circuitry. Advances in synthesis, characterization, and modeling in recent decades have enabled the exploration of metal nanostructures in catalysts, electrode materials, sensors, therapeutics, and electric circuits $^{1-4}$. Examples include surfactant-directed growth and assembly of metal nanostructures ${ }^{5-9}$, electrocatalysts in water splitting and fuel cells $s^{3,10}$ binding of ligands, peptides, and proteins in sensors ${ }^{11,12}$, interactions of metal nanoparticle-based therapeutics with cells, synthesis of multidimensional metal nanostructures ${ }^{13}$ and remediation of the environment from toxic and radioactive metal species ${ }^{14}$. Interactions of metal nanoparticles with electrolytes, biomolecules, surfactants, and organic species play an important role and are often hard to quantify in experiments. Imaging the synthesis, material function, and tracking of nanoparticles at a sub-micrometer scale is difficult, and the location of ions, small molecules, and organic matter remain typically elusive in the time domain during assembly and surface reactions. The interpretation of experimental data, guidance in alloy composition, surface-surfactant combinations, and prediction of nanoscale behavior for specific applications can be significantly enhanced by simulations.

The earlier introduction of interpretable Lennard-Jones parameters for 8 face-centered cubic (fcc) elemental metals ${ }^{15}, \mathrm{Ag}, \mathrm{Al}$, $\mathrm{Au}, \mathrm{Cu}, \mathrm{Ni}, \mathrm{Pb}, \mathrm{Pd}$, and $\mathrm{Pt}$, has led to interesting insights and applications (Supplementary Figs. 1-3). The models accurately predict interactions of metal surfaces with solvents ${ }^{16}$, electrolytes, and recognition mechanisms of organic molecules (Supplementary Fig. 1a) ${ }^{17-20}$. The reliability is better than or equal to density functional calculations (DFT) calculations, including dispersion- corrected density functionals. The dynamics of complex interfaces can be studied in unprecedented accuracy due to many orders of magnitude lower computational cost, including quantitative predictions of crystal growth (Supplementary Fig. 1b, c) ${ }^{21-23}$, ligand exchange, and sensitive catalytic processes (Supplementary Fig. 1d ${ }^{24,25}$. Specific examples include electrode properties in complex electrolytes (Supplementary Fig. 1e) ${ }^{26}$, the dynamics of nanoparticles at oil/water interfaces (Supplementary Fig. 1f) ${ }^{27}$, mechanical properties of metal-polymer nanocomposites (Supplementary Fig. $1 \mathrm{~g})^{28}$, wettability of Pt surfaces as a function of curvature (Supplementary Fig. 1h), diffusion of nano-cars on metal surfaces (Supplementary Fig. S1i) ${ }^{29}$, strain fields and defects in core-shell nanoparticles (Supplementary Fig. $1 \mathrm{j})^{30}$, and properties of alloys (Supplementary Fig. $1 \mathrm{k})^{31}$. The models can be extended to bcc metal structures and incorporate polarization by external electric fields on the fly using extensions with virtual electrons (Supplementary Fig. 11 and Supplementary Fig. $3 m)^{16,32}$. More details and examples can be found in Supplementary Figs. 1-3. Specific correlations with experimental data include structural data (X-ray diffraction patterns, TEM, AFM, STM), spectroscopy (UV, IR, SFG, Raman, NMR, XPS), binding constants, defect energies, observations by QCM, DSC, I/V curves, voltammetry, and turnover frequencies in catalysis (Supplementary Figs. 1-3). Overall, the models have explained a wide range of experimental data for metals, electrolyte, and organic interfaces, and can be used for quantitative predictions.

In this paper, we introduce and validate Lennard-Jones (LJ) parameters for ten further fcc metals in the periodic table and for stainless steel, including Ac, Ca (a), Ce ( $\gamma)$, Es ( $\beta)$, Fe ( $(\gamma)$, Ir, Rh, Sr (a), $\mathrm{Th}(\mathrm{a})$, and $\mathrm{Yb}(\beta)$. Consistent $\mathrm{L}$ parameters for these additional metals expand the space of accessible alloy compositions

${ }^{1}$ Department of Chemical and Biological Engineering, University of Colorado at Boulder, Boulder, CO 80301, USA. ${ }^{2}$ Departments of Biological Sciences, Chemistry, and Bioengineering, Lehigh University, Bethlehem, PA 18015, USA. ${ }^{\circledR}$ email: hendrik.heinz@colorado.edu 
exponentially ${ }^{31}$, including high entropy alloys and metallic glasses ${ }^{33,34}$. Among the metals covered here, prior $\mathrm{L}$ parameters were only available for iron and have large errors (Supplementary Table 1). Iron, which forms a bcc crystal under ambient conditions, assumes an fcc structure at high temperature and in austenitic steels. Embedded atom models (EAMs) are available for 7 out of the 10 metals $^{34}$, and density functional theory (DFT) calculations are possible for all metals with some limitations for the heaviest elements (Ac, Es, Th, Yb).

Simulations of metal nanostructures to-date have been mainly carried out using quantum-mechanical methods, LJ parameters, embedded atom models, and specialized potentials. In the following, we summarize advantages and limitations. (1) Quantum-mechanical methods include tight binding and DFT, which can be applied up to $\sim 10^{3}$ atoms. Unique information includes the localization of electrons on atoms and relationships to a broad range of physical properties. For example, atomic positions, electron density maps, band structure, bulk properties, and chemical reactions can be studied without limitations on chemical composition. Restrictions include the small system size, limited dynamics (few picoseconds), problems with reliability, and high computational expense ${ }^{35-37}$. Deviations of computed lattice parameters, surface energies, and mechanical properties from experimental data can be high and range from 2 to $100 \%$. The uncertainties arise from assumptions in exchange-correlation functionals, fit parameters for nonbonded interactions, and relativistic effects by $d$ and $f$ electrons. In particular, metal surface energies often deviate by $50 \%$ from experimental data using common density functionals in DFT calculations, and errors in excess of $100 \%$ are known for the adsorption energies of rare gases and other molecules ${ }^{36-38}$. The errors can be reduced to the $10-50 \%$ range by dispersion-corrected functionals (Grimme D3, optPBE, Scheffler), however, significant uncertainties remain ${ }^{36,37,39}$. Another question from a user perspective is: which density functional should one choose? Many available options yield divergent results for well-known experimental data, and tradeoffs for one property versus another are common $36,37,40$. Overall, predictions of surface, interfacial, and mechanical properties are approximate and require validation by experimental data. DFT data are therefore only of qualitative use to inform force fields, e.g., the Interface force field (IFF) ${ }^{15,16,41}$, and EAMs ${ }^{34}$.

(2) Lennard-Jones (LJ) potentials can be used for up to $\sim 10^{8}$ atoms and dynamic processes beyond microseconds, and are computationally about $10^{8}$ times less expensive than quantummechanical methods ${ }^{42}$. LJ parameters match lattice parameters in $<0.1 \%$ deviation from experiment and surface and interfacial energies in $<5 \%$ deviation from the experiment, which is one order of magnitude more accurate than DFT and EAMs. LJ models are compatible with numerous force fields designed for biomolecules and organic species that include $\mathrm{LJ}$ potentials ${ }^{44-50}$. Mechanical properties deviate similar to quantum methods (about $25 \%)$, in some cases less $(0 \%)$ or more $(100 \%)$ depending on the Poisson ratio of the metal (quantitative fit when 0.36-0.37). LJ parameters are simple, with 2 parameters per metal, and have a clear physical interpretation. They can be extended for features of the electronic structure to improve mechanical properties, capture image potentials of adsorbed ions and external electric fields on the fly ${ }^{16,32}$, and be integrated into modeling of chemical reactions.

(3) Embedded atom models (EAM) and modified embedded atom models (MEAM) are useful for metallic structures up to $\sim 10^{5}$ atoms and simulation times of nanoseconds, excluding aqueous and organic interfaces ${ }^{34,51,52}$. The computational expense of EAMs is $\sim 10^{5}$ times lower compared to DFT and $\sim 10^{3}$ times higher than that of $\mathrm{LJ}$ potentials. The reliability of computed structural, thermal, and mechanical properties can be excellent with 0.1 to $5 \%$ error relative to experiments, clearly better than with DFT methods ${ }^{34}$. Surface energies are computed too low by up to $50 \%$, i.e., up to ten times larger error compared to $L J$ potentials and experimental data ${ }^{30,34,52}$. Mechanical properties can be excellent with $\sim 5 \%$ deviation and better than $L$ potentials ${ }^{34}$, while deviations up to $40 \%$ from experiments are also found depending on the $\mathrm{EAM}^{30,52}$. On balance, lattice parameters, surface properties, and mechanical properties are of better reliability than tight binding quantum-mechanical methods ${ }^{34}$. Shortcomings include the difficulty to simulate electrolyte, organic, and biomolecular interfaces due to lack of compatibility with existing bonded force fields and high errors in surface energies ${ }^{53}$. EAM potentials also need refitting for alloys due to 20 to 40 fit parameters per metal with low or no interpretability ${ }^{34}$.

(4) Some specialized potentials for metal-aqueous and metal-organic interfaces have been proposed ${ }^{54-56}$. The accuracy and utility is typically lower than for LJ potentials at a similar computational cost. For example, GolP-CHARMM ${ }^{56}$ requires atom positions to be fixed and the validation of lattice parameters, surface energies, and interfacial energies is challenging. The number of fit parameters tends to be high and specific interaction parameters with other species need to be derived for every metal, as well as for every individual ( $h k l)$ surface of a metal. In comparison, LJ parameters function in high accuracy using standard combination rules and need only 2 parameters per metal including all interfaces.

In summary, LJ parameters have significant advantages over other models and can be used in conjunction with QM and EAMs when helpful. In the following, we describe the $L J$ functional forms, parameters, validation, and example applications to aqueous interfaces, iron alloys, and surface reactions. The paper ends with a discussion and computational methods. The Supplementary Information contains complete details of computational methods (Supplementary Methods), further discussion (Supplementary Notes 1-7), as well as a Supplementary Dataset including unit cells and surface models of all metals, as well as force field files and run scripts for simulations.

\section{RESULTS}

\section{Functional form and interpretation of the $\mathrm{L}$ parameters}

Lennard-Jones potentials are often used in the 12-6 and 9-6 forms and with other combinations of exponents ${ }^{41,57,58}$. For example, the force fields AMBER ${ }^{47}, \mathrm{CHARMM}^{45}, \mathrm{CVFF}^{43}$, DREIDING $^{48}$, and OPLS-AA ${ }^{46}$ use a $12-6 \mathrm{LJ}$ potential:

$$
E=\varepsilon_{0}\left[\left(\frac{\sigma}{r}\right)^{12}-2\left(\frac{\sigma}{r}\right)^{6}\right]=\frac{A}{r^{12}}-\frac{B}{r^{6}}\left(\text { with } A=\varepsilon_{0} \sigma^{12}, B=2 \varepsilon_{0} \sigma^{6}, \sigma=\sqrt[6]{\frac{2 A}{B}}\right)
$$

The force fields COMPASS $^{49}, \mathrm{CFF}$, and PCFF employ a 9-6 LJ potential $^{44}$ :

$E=\varepsilon_{0}\left[2\left(\frac{\sigma}{r}\right)^{9}-3\left(\frac{\sigma}{r}\right)^{6}\right]$

In Eqs. (1) and (2), $\sigma$ represents the equilibrium non-bond distance between two atoms of the same type and $\varepsilon_{0}$ the energy at the equilibrium distance. In systems with multiple atom types, such as alloys and metal-electrolyte interfaces, the parameters $\varepsilon_{0, i j}$ and $\sigma_{i j}$ represent non-bond energies between different atom types $i$ and $j$ and are obtained by combination rules.

Accordingly, every homoatomic fcc metal is described by two parameters $\sigma$ (formerly called $r_{0}{ }^{15}$ ) and $\varepsilon_{0} . \sigma$ represents the density and $\varepsilon_{0}$ the surface energy under standard conditions, respectively. The density is a structural quantity and the surface energy an energy quantity, which together capture the basic function of a Hamiltonian to reproduce structures and energies at a given reference state ${ }^{41,53}$. L models, and force fields more broadly, do not cover a temperature range of several thousand Kelvin or extreme pressures without adjustments. Therefore, a reference state is important, for which we use a temperature of $298.15 \mathrm{~K}$ and 
a pressure of $101.325 \mathrm{kPa}$ (contrary to $0 \mathrm{~K}$ in DFT databases) ${ }^{15,41}$. This reference state is also used in thermochemical tables, thermodynamic tables, and in IFF' ${ }^{41}$. We discourage the common use of cohesive energies to calibrate $\varepsilon_{0}$ for solids and liquids with high boiling points, which can cause up to hundredfold errors in surface and interfacial properties, in favor of surface energies at 298.15 K. Cohesive energies imply a phase transition to metal vapor at the boiling point (up to $4000 \mathrm{~K}$ ) and usually create a conflict in the reference state, making them unsuitable for model validation (Supplementary Note 1). Key properties of a Hamiltonian, i. e., lattice parameters and surface energy at a well-defined temperature and pressure, enable the consistent definition and validation of force fields (as well as of density functionals and EAMs) ${ }^{41}$.

We thus used experimental data for the density ${ }^{59}$ and the surface energy ${ }^{60-62}$ under standard conditions to assign $\sigma$ and $\varepsilon_{0}$ for each metal to maximize interpretability, reliability, and compatibility ${ }^{15}$. The $L$ models yield surface energies of other crystal faces in agreement with experimental data, reproduce the monolayer hydration energy with water within $\sim 5 \%{ }^{16}$, as well as interactions with electrolytes and adsorption of organic molecules without further fit parameters (5-10\% error depending on the quality of the non-metal parameters) ${ }^{17-19,21,22,63}$. Mechanical properties are approximate due to the simplicity of the twoparameter model, which leads to a constant ratio of the elastic constants $C_{12} / C_{44}=1$. The performance ranges from excellent to poor depending on the proximity of the Poisson ratio of the metal to the ideal Poisson ratio of the $L J$ potentials $(\sim 0.36$ for 12-6 $\mathrm{LJ}$ potentials and $\sim 0.37$ for 9-6 LJ potentials).

\section{Integration of the $\mathbf{L}$ parameters into other force fields}

The two parameters $\sigma$ and $\varepsilon_{0}$ can be imported into any force field that uses an LJ potential using standard combination rules. No change in LJ parameters is required since our validation focuses on the properties of the elemental fcc metals ${ }^{15}$. Therefore, the same density and the same surface energy are obtained upon incorporation into any other force field regardless of combination rules.

Physically justified, standard combination rules such as in AMBER, CHARMM, OPLS-AA, CVFF, DREIDING, or PCFF perform well to derive non-bond interactions $\varepsilon_{0, i j}$ and $\sigma_{i j}$ between different atom types $i$ and $j$ without the addition of further parameters. In this manner, a wide range of existing parameters for inorganic, organic, and biological compounds can be used to simulate metalhybrid materials and interfaces. The major factor that determines the accuracy for combined systems is the quality of the parameters for the other (non-metal) species, i.e., to which extent they follow the same validation of structures and energies near the reference state as in IFF $(298.15 \mathrm{~K}$ and $101.3 \mathrm{kPa})$. Excellent accuracy for metal-water, metal-organic, and other interfaces has been previously shown ${ }^{16,41,64-66}$. By analogy, biomolecular force fields rely on identical combination rules to simulate the hydration of ions and proteins using distinct atom types in water, ions, and proteins.

In this paper, we used default combination rules for CVFF (12-6 $\mathrm{L}$ potential) and PCFF (9-6 LJ potential) and standard water models (Supplementary Note 2).

\section{LJ parameters for FCC metals}

The newly developed 12-6 and 9-6 LJ parameters are listed in Table 1. The metals are represented by charge-neutral atoms with repulsive and dispersive van-der-Waals interactions following Eqs. (1) and (2), respectively. Lattice parameters and densities from X-ray data ${ }^{59}$, as well as surface energies of the low energy (111) surfaces ${ }^{60-62}$, were employed to assign the parameters $\sigma$ and $\varepsilon_{0}$ at $298 \mathrm{~K}$ and $101.3 \mathrm{kPa}$ (Fig. 1a, b). The two parameters have only a small interdependence which reflects the physically distinct roles.
Table 1. 12-6 and 9-6 Lennard-Jones parameters for $10 \mathrm{fcc}$ metals, including non-bond diameters $\sigma$ and well depths $\varepsilon_{0}$, as well as constants $A$ and $B$ (in case of 12-6 LJ parameters).

\begin{tabular}{|c|c|c|c|c|c|c|}
\hline Metal & $\begin{array}{l}12-6 \\
\llcorner J \sigma \\
(\AA)^{\mathrm{a}}\end{array}$ & $\begin{array}{l}12-6 \mathrm{LJ} \varepsilon_{0} \\
(\mathrm{kcal} \\
\left.\mathrm{mol}^{-1}\right)^{\mathrm{a}}\end{array}$ & $\begin{array}{l}12-6 \mathrm{LJ} A(\mathrm{kcal} \\
\left.\mathrm{mol}^{-1} \AA^{12}\right)^{\mathrm{a}}\end{array}$ & $\begin{array}{l}12-6 \mathrm{LJ} B \\
(\mathrm{kcal} \\
\mathrm{mol}^{-1} \\
\left.\AA^{6}\right)^{\mathrm{a}}\end{array}$ & $\begin{array}{l}9-6 \mathrm{LJ} \\
\sigma(\AA)^{\mathrm{b}}\end{array}$ & $\begin{array}{l}\text { 9-6 } \mathrm{LJ} \varepsilon_{0} \\
(\mathrm{kcal} \\
\left.\mathrm{mol}^{-1}\right)^{\mathrm{b}}\end{array}$ \\
\hline$A C$ & 3.843 & 6.51 & $67,550,000$ & 41,940 & 3.907 & 5.40 \\
\hline $\mathrm{Ca}(\alpha)$ & 4.025 & 3.36 & $60,750,000$ & 28,570 & 4.088 & 2.80 \\
\hline $\mathrm{Ce}(\gamma)$ & 3.734 & 6.38 & $46,870,000$ & 34,590 & 3.797 & 5.28 \\
\hline Es $(\beta)$ & 4.133 & 2.88 & $71,540,000$ & 28,710 & 4.195 & 2.39 \\
\hline Ir & 2.785 & 9.20 & $2,003,000$ & 8586 & 2.836 & 7.48 \\
\hline Fe $(\gamma)^{c}$ & 2.590 & 6.00 & 546,700 & 3620 & 2.645 & 4.79 \\
\hline $\mathrm{Rh}$ & 2.757 & 7.84 & $1,512,000$ & 6886 & 2.807 & 6.38 \\
\hline $\operatorname{Sr}(\alpha)$ & 4.379 & 3.40 & $169,000,000$ & 47,950 & 4.445 & 2.85 \\
\hline Th $(\alpha)$ & 3.683 & 8.47 & $52,760,000$ & 42,280 & 3.746 & 7.01 \\
\hline $\mathrm{Yb}(\beta)$ & 3.942 & 2.71 & $38,160,000$ & 20,340 & 4.001 & 2.24 \\
\hline
\end{tabular}

aUsing the 12-6 Lennard-Jones potential according to Eq. (1), including the force fields AMBER, CHARMM, CVFF, DREIDING, and OPLS-AA.

bUsing the 9-6 Lennard-Jones potential according to Eq. (2), including the force fields CFF, PCFF, and COMPASS.

'Parameters are given for fcc $\gamma$-Fe in stainless steel alloys and can be used for bcc $\alpha$-Fe. In the simulation of bcc $\alpha-\mathrm{Fe}$, atomic positions should be fixed to prevent a transition into fcc $\gamma$-Fe.

The values were optimized by a least-squares fit and include only significant digits necessary to achieve computational results well within the experimental uncertainty. The difference between 12-6 and 9-6 LJ parameters for a given metal is characterized by an average increase in the values for $\sigma$ by $\sim 1.7 \%$ and an average decrease in the values for $\varepsilon_{0}$ by $\sim 18 \%$ towards the 9- $6 \mathrm{LJ}$ potential. The differences reflect weaker repulsion and stronger attraction in the 9-6 $\mathrm{L}$ potential relative to the $12-6 \mathrm{~L}$ potential (Table 1) ${ }^{15,67}$. Further validation included water interfacial energies and mechanical properties (Fig. 1a-e). The interpretation is straightforward: geometry $(\sigma)$ and surface energy $\left(\varepsilon_{0}\right)$ of the metals determine the interaction with other compounds and are incorporated in the highest possible accuracy ${ }^{41,67}$. Atomistic models of variously shaped metal nanostructures and input files for simulations can be built using any suitable programs, e.g., Materials Studio, LAMMPS, CHARMM and GROMACS tools. Specifically, the Nanomaterial Modeler module in CHARMM-GUI allows to build customized ( $h k l)$ models and interfaces of metals, and the generation of simulation input in various file formats (Supplementary Fig. 4 and Supplementary Note 3) ${ }^{68,69}$.

The models can be applied in a temperature range of $298 \mathrm{~K} \pm$ $200 \mathrm{~K}$ for the highest accuracy, and very good results outside this window such as melting points have also been reported ${ }^{70}$. Modifications of the $L J$ parameters for significantly different temperatures and pressures can be made by adjustment of $\sigma$ and $\varepsilon_{0}$ (Supplementary Note 4).

\section{Lattice parameters}

The lattice parameters and density, respectively, were computed by molecular dynamics simulations in the NPT ensemble for systems of more than $1.8 \mathrm{~nm}$ side length (Fig. 1a and Supplementary Methods). Computed lattice parameters typically agree with experimental data with less than $0.05 \%$ deviation (Table 2 ). Excellent agreement is expected as the two parameters $\sigma$ and $\varepsilon_{0}$ were fitted to the density of each metal. The deviations under $0.05 \%$ are a factor 10 smaller compared to DFT calculations and comparable to EAMs. As a result of the minor deviations in lattice parameters $\Delta a$, deviations in densities $\Delta \rho$ under standard 
a

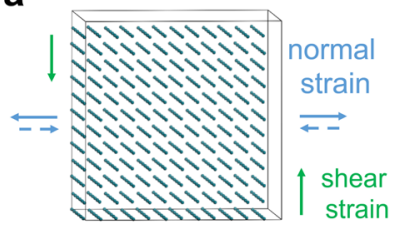

(100) boundaries b

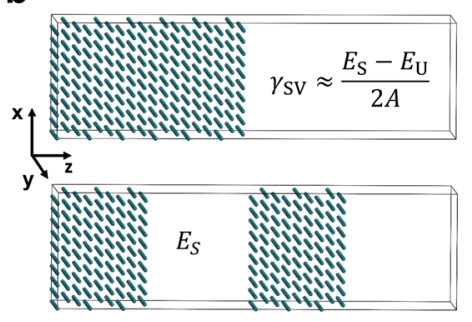

C $E_{S L}$

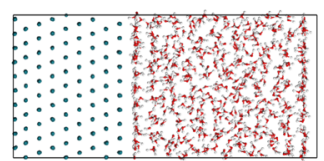

(111) metal-water interface
$E_{S}$

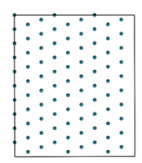

pure metal
$E_{L}$

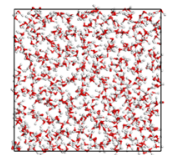

pure

water

$$
\gamma_{S L}=\frac{E_{S L}-E_{L}-E_{S}}{2 A}-\frac{T \Delta S}{2 A}
$$
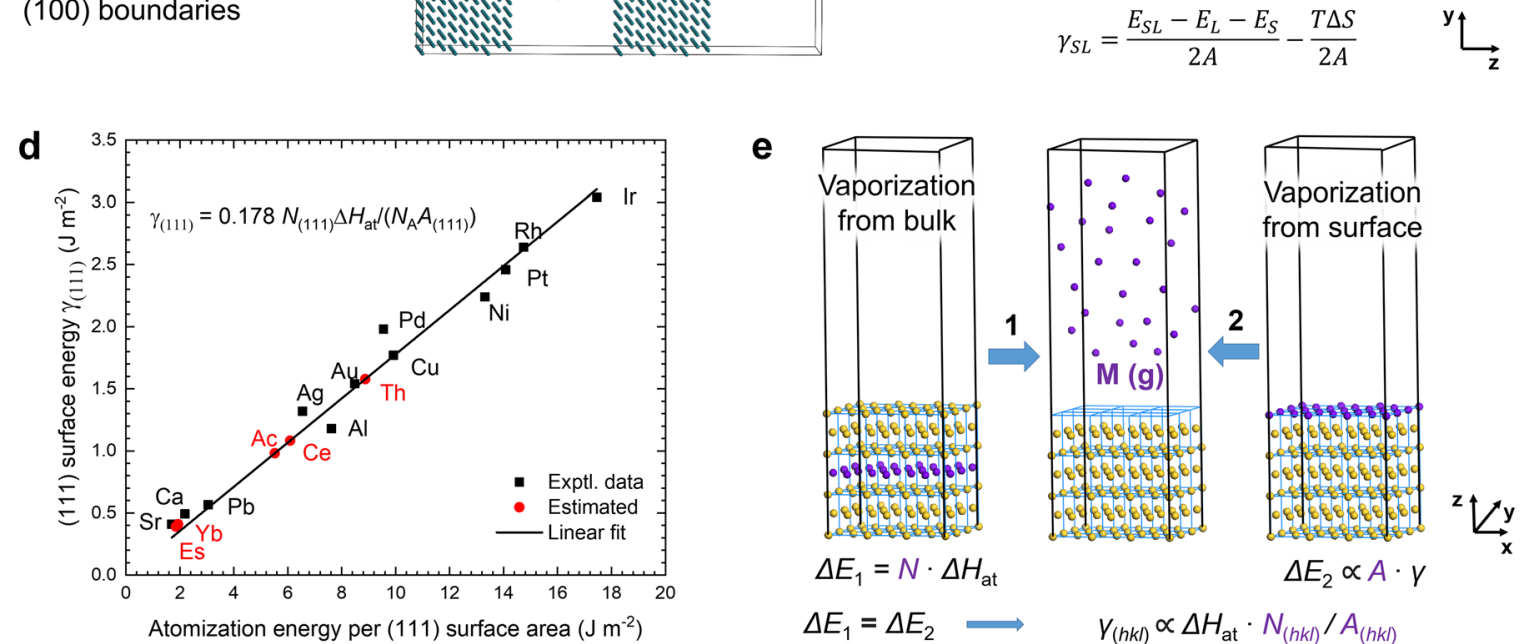

Fig. 1 Calculation of the physical properties in molecular dynamics simulation. a Illustration of the calculation of lattice parameters and mechanical properties of the metals using a cubic $6 \times 6 \times 6$ supercell of Rh (side length $2.282 \mathrm{~nm}, 864$ atoms). The direction of normal strain (blue arrows) and shear strain (green arrows) for the calculation of mechanical properties is indicated. Solid blue arrows $=$ tension, dashed blue arrows = compression. b Illustration of the calculation of the (111) surface energy. (100), (111), and (110) cleavage planes can be generated perpendicular to the vertical axis using the model in a and using equivalent orthorhombic supercells (Supplementary Dataset and refs. $\left.{ }^{41,96}\right)$. Two boxes with unified and separate surfaces were used, along with the corresponding average energies $E_{\mathrm{U}}$ and $E_{\mathrm{S}}$. $c$ Setup for the calculation of the (111) metal-water interfacial energy. Three boxes containing a metal-water interface $\left(E_{\mathrm{SL}}\right)$, pure water $\left(E_{\mathrm{L}}\right)$, and pure metal $\left(E_{S}\right)$ were used. d Linear relationship between surface energy and atomization energy. The (111) surface energy is proportional to the atomization energy normalized per (111) surface area for all fcc metals. The relationship was used to estimate the unknown surface energies for $\mathrm{Es}, \mathrm{Yb}, \mathrm{Ac}, \mathrm{Ce}$, and Th (red data points). e Derivation of the relation in d. Atomization of a layer of atoms from the bulk (process 1$)$ and removal of a layer of surface atoms (process 2) are thermodynamically equivalent. Process 1 describes atomization. The equivalent process 2 creates a new surface of energy $\gamma$, plus it disconnects all bonds between neighbor atoms in the detached atomic layer. These additional contributions lead to an atomization energy per surface area that is several times higher than the surface energy (see $x$ axis versus $y$ axis in d).

Table 2. Lattice parameters for $(5 \times a)^{3}$ fcc supercells according to Xray diffraction data from experiments and molecular dynamics simulations at $298 \mathrm{~K}$ and atmospheric pressure in the NPT ensemble (in $\AA$ ).

\begin{tabular}{|c|c|c|c|}
\hline Metal & $\operatorname{Expt}^{\mathrm{a}}$ & $12-6 \mathrm{LJ}$ & $9-6 \mathrm{LJ}$ \\
\hline Ac & 26.555 & 26.559 & 26.543 \\
\hline $\mathrm{Ca}(\alpha)$ & 27.942 & 27.947 & 27.953 \\
\hline $\mathrm{Ce}(\gamma)$ & 25.805 & 25.805 & 25.796 \\
\hline Es $(\beta)$ & 28.750 & 28.768 & 28.747 \\
\hline $\mathrm{Fe}(\gamma)(1189 \mathrm{~K})^{\mathrm{b}}$ & 18.197 & 18.161 & 18.300 \\
\hline Ir & 19.196 & 19.203 & 19.199 \\
\hline $\mathrm{Rh}$ & 19.016 & 19.016 & 19.014 \\
\hline $\operatorname{Sr}(\alpha)$ & 30.420 & 30.423 & 30.421 \\
\hline Th $(\alpha)$ & 25.421 & 25.423 & 25.424 \\
\hline $\mathrm{Yb}(\beta)$ & 27.424 & 27.431 & 27.425 \\
\hline Std dev to expt (excl $\gamma-\mathrm{Fe})$ & \pm 0.001 & \pm 0.003 & \pm 0.004 \\
\hline \multicolumn{4}{|c|}{$\begin{array}{l}\text { aRef. }{ }^{59} \text {. } \\
\text { bLattice parameters for } \gamma \text {-iron (fcc) were calculated at } 1189 \mathrm{~K} \text { for a stable fcc } \\
\text { crystal structure } e^{98} \text {. Computed lattice parameters as a function of } \\
\text { temperature from } 0 \text { to } 1661 \mathrm{~K} \text { are given for } \alpha \text {-iron (bcc) and } \gamma \text {-iron (fcc) } \\
\text { in Supplementary Table } 2 \text {. }\end{array}$} \\
\hline
\end{tabular}

conditions are typically $<0.1 \%\left(\Delta \rho \sim\left[1-(1-\Delta a / a)^{3}\right]\right)$. Iron features a slightly higher deviation up to $0.5 \%$ in lattice parameters due to a different reference state (derived at $1189 \mathrm{~K}$ for fcc $\mathrm{Y}$-iron and then used at $298 \mathrm{~K}$ for bcc a-iron and fcc steel alloys).

\section{Surface energy}

The key advantages of the $L J$ models are accurate surface and interfacial energies. (111) surface energies are fitted to experimental data within the experimental uncertainty of $\pm 1- \pm 5 \%$ (Figs. 1b, 2a, and Table 3). Experimental data for surface energies in high quality are available from Tyson and Miller ${ }^{60}$, Keene ${ }^{61}$, and Mills ${ }^{62}$. The data are further backed by a correlation with well-known atomization energies (Fig. 1d, e) ${ }^{59}$. Surface energies and surface free energies are equal within the experimental uncertainty since entropy contributions to the surface free energies are less than $-0.01 \mathrm{~J} \mathrm{~m}^{-2}$ (Supplementary Methods) ${ }^{15}$. Alternative models such as EAM potentials ${ }^{34,51,52}$ and density functionals ${ }^{35,71}$ perform typically poorly for surface energies and have deviations up to $\sim 50 \%$ (Table 3). Calculations using density functionals are also more difficult for heavy elements such as Ac, Ce, and Es (Table 3). Discrepancies in surface energies directly and proportionally affect the analysis of adsorption of molecules, assembly, and stability of nanostructures, as well as catalytic processes on surfaces ${ }^{21,25,36-38}$. Computed adsorption energies of simple molecules such as noble 


\section{a}

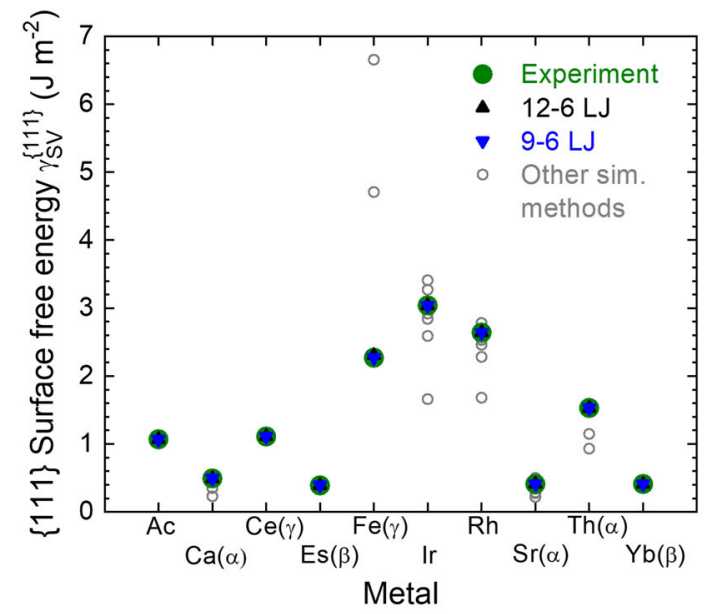

b

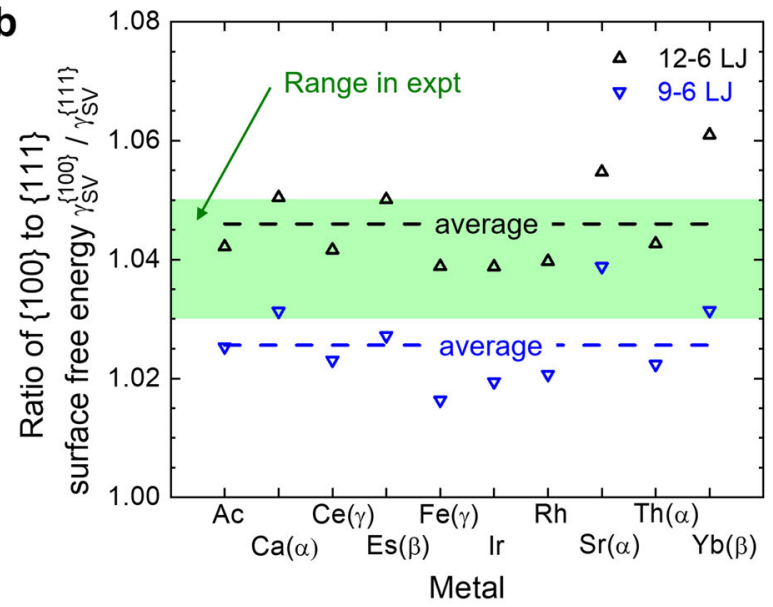

C

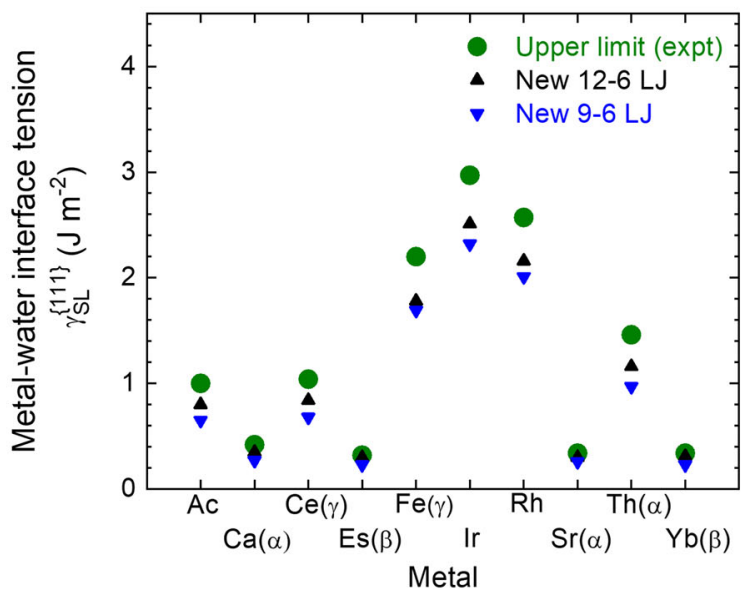

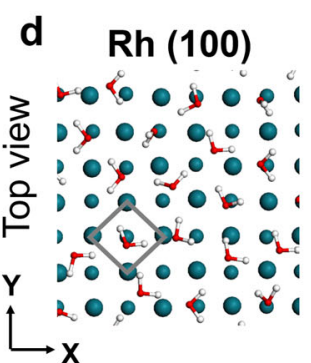

e

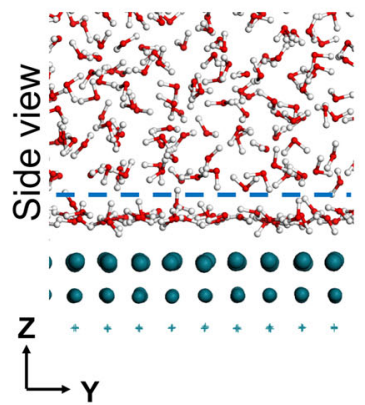

f

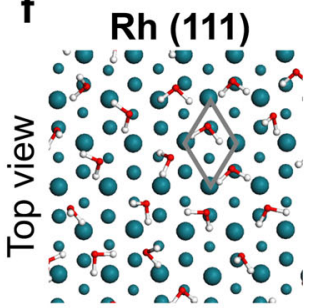

g

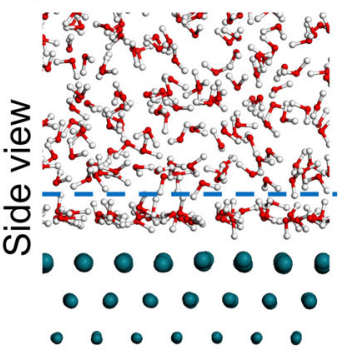

h $\quad \operatorname{Sr}(100)$
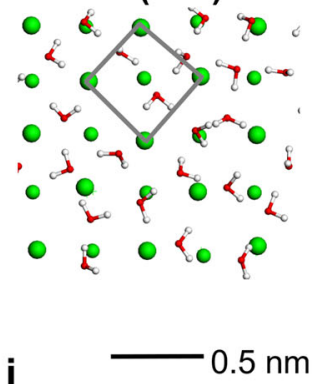

\%

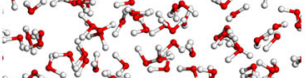

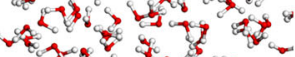
a
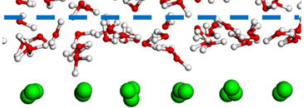

- 0 - 0

$1 \mathrm{~nm}$
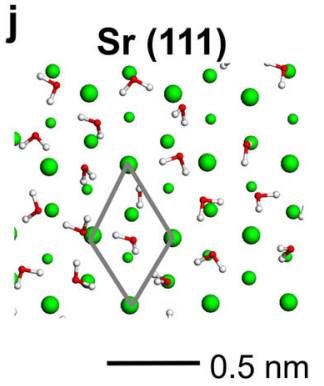

k

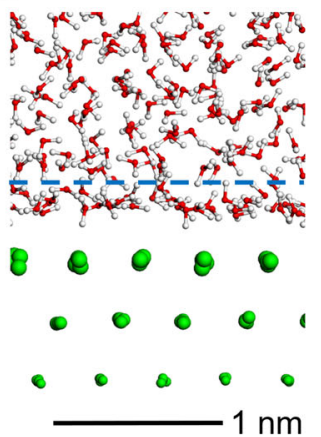

Fig. 2 Surface and interfacial properties of the fcc metals. a The (111) surface free energy in experiments, in the simulation with the $L J$ models, and according to other computational models such as DFT, tight binding, and EAM. The LJ models achieve the best match. $\mathbf{b}$ Ratio of the $(100)$ surface free energy to the (111) surface free energy using the $L$ models. Experiments suggest values between 1.03 and 1.05 consistent with the calculations using the $L J$ parameters (refs. ${ }^{73-75}$ ). c The (111) metal-water interface tensions computed with the $12-6 \mathrm{LJ}$ models (CVFF, CHARMM) and 9-6 LJ models (PCFF). Flexible SPC and SPC-like water models were used. The upper limit of the metal-water interface tension according to experimental data and the Young equation is also shown, showing agreement with the computed data. d, e Example of a Rh (100)-water interface in equilibrium. About 1 water molecule in the first molecular layer is available per $1 \mathrm{Rh}$ atom in the top atomic layer, and the first molecular layer of water on the surface is strongly bound. $\mathbf{f}$, $\mathbf{g}$ Similar trends are seen on an Rh (111)-water interface in a different geometry. Water molecules are frequently found near epitaxial sites (fcc and hcp) and less often near top sites on both (111) and (100) surfaces. $\mathbf{h}$, i A Sr (100)-water interface before the reaction to $\mathrm{Sr}(\mathrm{OH})_{2}$ and $\mathrm{H}_{2}$. Due to a larger lattice constant of Sr compared to $\mathrm{Rh}$, much wider spaces between metal surface atoms are seen, allowing partial water penetration. Approximately 2 water molecules in the first molecular layer are available per $1 \mathrm{Sr}$ atom in the top atomic layer. The first molecular layer of water is less structured due to the lower surface energy of Sr compared to Rh, and water molecules exchange faster. $\mathbf{j}$, k Similar trends are seen on the (111) surfaces in a different geometry. 


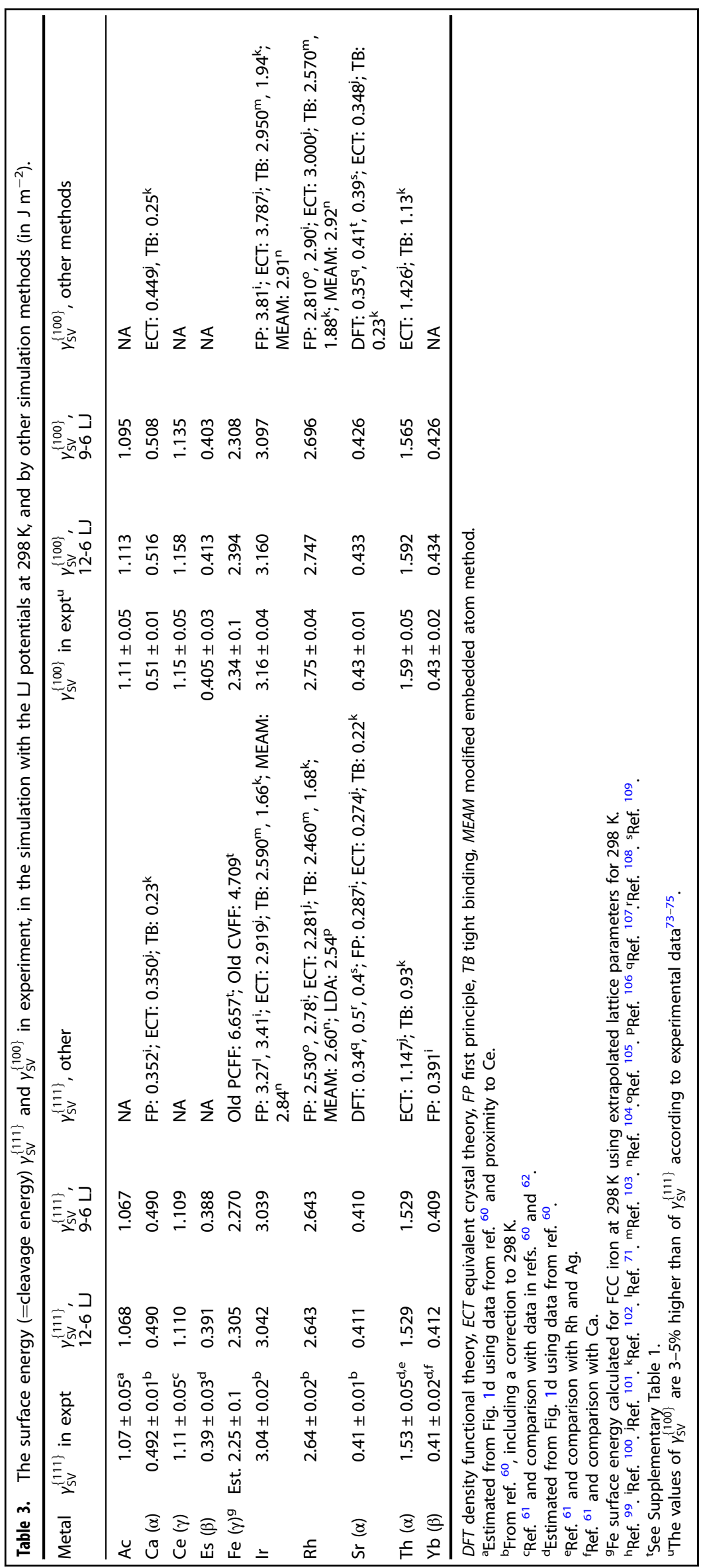


gases and organic molecules using DFT models deviate up to several $100 \%$ from experimental data due to compounding of errors for the metals and for the non-metal molecules ${ }^{36-38}$. EAMs cannot be easily used to simulate metal-organic interfaces and would face similar challenges. In comparison, $L J$ parameters and standard combination rules, for example, using IFF, AMBER, CHARMM, OPLS-AA, or PCFF, reproduce the adsorption of water and organic molecules within $5-10 \%$ of experimental data without additional fit parameters, and are $10^{8}$ times less computeintensive than DFT calculations ${ }^{16,72}$.

For the (100) surface, computed surface energies are on average $4.7 \%$ or $2.7 \%$ higher than for the (111) surface, depending on whether $12-6$ or 9-6 LJ potentials are used, respectively (Table 3 and Fig. 2b). The different ratios reflect intrinsic differences due to the functional form of the LJ potential. Experimental measurements of the surface energy anisotropy, using information on nanoparticle shapes and facet stability, suggest 3-5\% higher surface energies for (100) surfaces relative to (111) surfaces ${ }^{73-75}$. Both types of $\mathrm{L}$ potentials reproduce the data in the right range and suggest that relative energies of $(h k l)$ surfaces mainly result from changes in surface geometry.

For some heavier metals including $\mathrm{Th}, \mathrm{Ce}, \mathrm{Ac}, \mathrm{Yb}$, and $\mathrm{Es}$, experimental data for surface energies were not available. We utilized a relationship between well-known atomization energies $\Delta H_{\text {at }}$ and surface energies $\gamma$ to obtain the missing data ${ }^{76}$. Surface energies can be considered a form of cohesive energy related to a (hkl) surface area (Fig. 1d, e). Division of the known atomization $\Delta H_{\text {at }}$ energies for $N$ atoms, i.e., cohesive energies, by the square of the lattice constant yields a linear correlation with (111) surface energies for all fcc metals:

For fcc metals: $\gamma_{\mathrm{SV}}^{(111)} \sim \frac{N_{(111)} \Delta H_{\mathrm{at}}}{A_{(111)}}=\frac{2 \Delta H_{\mathrm{at}}}{(\sqrt{3} / 2) N_{\mathrm{A}} a^{2}}$

Hereby, $N=2$ metal atoms are found per unit area of a (111) surface $\left(A_{(111)}=(\sqrt{3} / 2) a^{2}\right)$. The calculation utilizes the atomization energy $\Delta H_{\mathrm{at}}$ in $\mathrm{kJ} \mathrm{mol}^{-1}$, the Avogadro constant $N_{\mathrm{A}}$, and the lattice parameter $a$. The proportionality constant further depends on the coordination number and pairwise bond strength of the atoms in the detached atomic layer (Process 2 in Fig. 1e) ${ }^{76}$. Using known surface energies, corresponding atomization energies, and lattice parameters for (111) surfaces of fcc metals, we obtain:

For fcc metals: $\gamma_{\mathrm{SV}}^{(111)}=0.178 \cdot \frac{2 \Delta H_{\mathrm{at}}}{(\sqrt{3} / 2) N_{\mathrm{A}} a^{2}}=0.308 \cdot \frac{\Delta H_{\mathrm{at}}}{N_{\mathrm{A}} a^{2}}$

The linear correlation in Eq. (4) has average deviations under $5 \%$ (Fig. 1d). Using the training curve and known atomization energies for Th, $\mathrm{Ce}, \mathrm{Ac}, \mathrm{Yb}$, and $\mathrm{Es}$, we obtained the corresponding surface free energies with $~ 5 \%$ uncertainty, which have not been available to-date (red data points in Fig. 1d and entries in Table 3). The estimation of surface energies from atomization energies and unit cell geometry can also be helpful for other ( $h k l)$ surfaces and lattice types (bcc, hcp).

\section{Water, electrolyte, and organic interfaces}

Experimental data have shown that polar as well as nonpolar liquids spread on clean metal surfaces with contact angles of $0^{\circ 77}$. Computed metal-water contact angles are $0^{\circ}$ for all $18 \mathrm{fcc}$ metals and agree with experiments. In addition, an upper bound of the values for the metal-water interface tension $\gamma^{\mathrm{SL}}$ can be obtained from the Young equation:

$\gamma^{\mathrm{SL}}+\gamma^{\mathrm{LV}} \cos \theta=\gamma^{\mathrm{SV}}$

wherein $\gamma^{\mathrm{SV}}$ is the metal surface free energy (Table 3), $\gamma^{\mathrm{LV}}=73 \mathrm{~mJ}$ $\mathrm{m}^{-2}$ the water surface tension ${ }^{59}$, and $\theta$ the contact angle of water. The metal-water interface tension $\gamma^{\mathrm{SL}}$ is then expected to have a
Table 4. The metal-water interface tension $\gamma_{S L}^{\{111\}}$, including upper limits from experimental data and values from the simulation (in $\mathrm{J} \mathrm{m}^{-2}$ ).

\begin{tabular}{lccc}
\hline Metal & $\begin{array}{c}\text { Upper limit of } \gamma_{\mathrm{SL}}^{\{111\}} \\
\text { from expt }^{\mathrm{a}}\end{array}$ & $\gamma_{\mathrm{SL}}^{\{111\}}, 12-6 \mathrm{LJ}^{\mathrm{b}, \mathrm{d}}$ & $\gamma_{\mathrm{SL}}^{\{111\}}, 9-6 \mathrm{LJ}^{\mathrm{c}, \mathrm{d}}$ \\
\hline $\mathrm{Ac}$ & $1.00 \pm 0.05$ & $0.80 \pm 0.01$ & $0.65 \pm 0.01$ \\
$\mathrm{Ca}(\alpha)$ & $0.42 \pm 0.01$ & $0.35 \pm 0.01$ & $0.27 \pm 0.01$ \\
$\mathrm{Ce}(\gamma)$ & $1.04 \pm 0.05$ & $0.84 \pm 0.01$ & $0.68 \pm 0.01$ \\
$\mathrm{Es}(\beta)$ & $0.32 \pm 0.03$ & $0.30 \pm 0.01$ & $0.23 \pm 0.01$ \\
$\mathrm{Fe}(\gamma)^{\mathrm{e}}$ & Est. $2.18 \pm 0.1$ & $1.78 \pm 0.01$ & $1.69 \pm 0.01$ \\
$\mathrm{Ir}$ & $2.97 \pm 0.02$ & $2.51 \pm 0.01$ & $2.32 \pm 0.01$ \\
$\mathrm{Rh}$ & $2.57 \pm 0.02$ & $2.16 \pm 0.01$ & $2.01 \pm 0.01$ \\
$\mathrm{Sr}(\alpha)$ & $0.34 \pm 0.01$ & $0.30 \pm 0.01$ & $0.26 \pm 0.01$ \\
$\mathrm{Th}(\alpha)$ & $1.46 \pm 0.05$ & $1.16 \pm 0.01$ & $0.97 \pm 0.01$ \\
$\mathrm{Yb}(\beta)$ & $0.34 \pm 0.02$ & $0.31 \pm 0.01$ & $0.23 \pm 0.01$ \\
\hline
\end{tabular}

${ }^{\mathrm{a}}$ From the Young equation $\gamma^{\mathrm{SL}}=\gamma^{\mathrm{SV}}-\gamma^{\mathrm{LV}}$ (Eq. (6)).

${ }^{b}$ Flexible SPC water model in CVFF.

'SPC-like water in PCFF.

${ }^{\mathrm{d}}$ Free energies are reported that consist of the computed energy contribution and an added entropy corrections of $+0.06 \mathrm{~J} \mathrm{~m}^{-2}$ for Ac, Ce, $\mathrm{Fe}, \mathrm{Ir}, \mathrm{Rh}$, Th, and $+0.04 \mathrm{~J} \mathrm{~m}^{-2}$ for $\mathrm{Ca}$, Es, Sr, Yb for both 12-6 and 9-6 $\mathrm{LJ}$ values (Fig. 1c and Supplementary Methods).

eValues for a hypothetical fcc structure of iron at room temperature.

value between zero and $\left(\gamma^{\mathrm{SV}}-\gamma^{\mathrm{LV}}\right)$, i. e.,

$0<\gamma^{\mathrm{SL}}<\gamma^{\mathrm{SV}}-\gamma^{\mathrm{LV}}$

Computed interface tensions concur with expectations according to Eq. (6) (Fig. 2c and Table 4). We utilized a flexible SPC water model (CVFF) and a flexible SPC-like water model (PCFF). The use of a TIP3P water model instead of the flexible SPC model leads to the same results with a few percent difference. The computed metal-water interfacial energies are, on average, around $80 \%$ of the theoretical maximum using the 12-6 $\mathrm{L}$ parameters and $\sim 60 \%$ of the theoretical maximum using the 9-6 $\mathrm{LJ}$ parameters. The ratio also depends on the lattice spacing of the metal. As recently shown for gold, computed hydration energies for monolayer coverage match experimental values within $\pm 5 \%$ using $12-6 \mathrm{LJ}$ parameters and standard combination rules ${ }^{16}$. Attractive polarization due to image charges on the atomically flat surfaces equals $-0.020 \pm 0.02 \mathrm{~J} \mathrm{~m}^{-2}$ in the presence of bulk water, which equals $\sim 2 \%$ of the interface tension ${ }^{16,63,78}$. Attractive polarization is thus near-negligible in polar solvents. However, adsorption of ions and single water molecules in the gas phase, as well as strong electric fields can add significant attraction ${ }^{16}$.

The models are thus useful to examine electrolyte interfaces in high reliability. As an example, we illustrate the structure of water on Rh surfaces, which have a high interfacial energy of $\sim 2.1 \mathrm{~J} \mathrm{~m}^{-2}$ and a small lattice parameter (Fig. $2 \mathrm{~d}-\mathrm{g}$ ), in comparison to $\mathrm{Sr}$ surfaces with a much lower interfacial energy of $\sim 0.3 \mathrm{~J} \mathrm{~m}^{-2}$ and a $50 \%$ wider lattice parameter (Fig. $2 \mathrm{~h}-\mathrm{k}$ ). The packing of water molecules relative to the metal surface atoms and the attraction to the metal surface exhibit major differences. The distinct layering of water molecules is visible on the Rh surfaces (Fig. 2e, g). Sr surfaces lead to a less regular distribution of water molecules, which shows more similarity to the bulk structure of water (Fig. 2i, k). Differential dynamics at the metal-water interface has implications on surface contact of electrolytes, dissolved molecules and polymers, and surface reactivity.

Energies of adsorption and assembly of organic and biological molecules at metal surfaces have been shown to agree within $\pm 10 \%$ of experimental data $53,79,80$. Many examples using the existing $\mathrm{LJ}$ models for $8 \mathrm{fcc}$ metals are known (Supplementary 

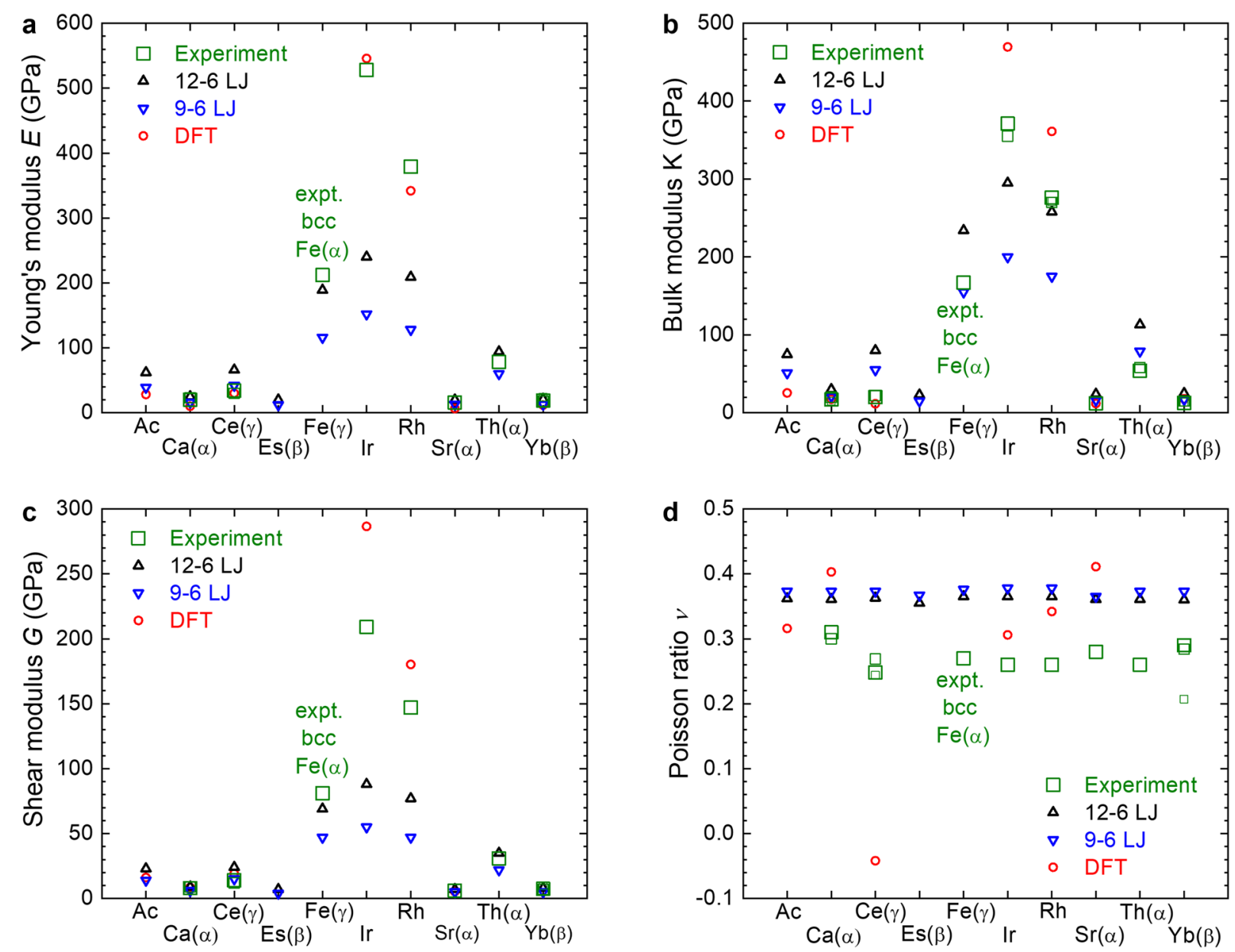

Fig. 3 Mechanical properties of the fcc metals. Data from the experiment, computation with the LJ models, and DFT are shown (with the RBPE functional where possible). a Young's modulus, b bulk modulus, $\mathbf{c}$ shear modulus, $\mathbf{d}$ Poisson ratios. Deviations of computed values from experiment are significant and tend to be lower with the 12-6 $\mathrm{LJ}$ potential.

Figs. 1-3). The reliability is up to an order of magnitude higher than with density functional methods (up to $100 \%$ scatter) ${ }^{36-38}$. Additional benefits are low computational cost, dynamics up to microsecond time scales, full electrolyte conditions, and length scales larger than other all-atom models (EAM). Effects of grain boundaries, ionic strength, solution $\mathrm{pH}$, dynamics of adsorbed proteins, polymers, and interactions of metal nanostructures with cell surfaces can be studied. The models are very well suited for large-scale computational screening and machine learning. The remaining minor uncertainties are associated with assumptions in the force field, such as a residual error in surface energies, differences using 12-6 $\mathrm{L}$ versus 9-6 $\mathrm{L}$ parameters, combination rules of the $L$ parameters, and the quality of the non-metal parameters.

\section{Mechanical properties}

Computed isotropic elastic properties agree qualitatively with experimental data, including Young's modulus, bulk modulus, shear modulus, and Poisson ratio (Fig. 3, Table 5, and Supplementary Tables 3 and 4). The correlation ranges from quantitative when the Poisson ratio of the metal is close to the ideal Poisson ratio of $L J$ potentials of 0.36 , to large deviations of $100 \%$ when the Poisson ratio is as small as $0.24-0.26$. This trend is the same for all $18 \mathrm{fcc}$ metals, i.e., including the $8 \mathrm{fcc}$ metals reported earlier (Supplementary Table 5$)^{15}$. The changes in mechanical properties as a function of the Poisson ratio, while the fcc crystal structure is the same, suggest an association with the specific electronic structure of each metal. The 12-6 LJ potential often fares better than the 9-6 $L$ potential, with average deviations of $+11 \pm 53 \%$ in Young's modulus, $87 \pm 98 \%$ in bulk modulus, $+1 \pm 47 \%$ in shear modulus, and $+35 \pm 8 \%$ in Poisson ratio relative to experiment (Table 5). The 9-6 $\mathrm{LJ}$ potential yields average errors of $-30 \pm 34 \%$ in Young's modulus, $+28 \pm 68 \%$ in bulk modulus, $-35 \pm 30 \%$ in shear modulus, and $+39 \pm 9 \%$ in Poisson ratio. The scatter with the 9-6 $\mathrm{L}$ potential is somewhat lower although it tends to systematically underestimate elastic moduli by up to $-30 \%$. The calculations were also carried out with different molecular dynamics codes, including Discover and LAMMPS, and results are identical within a few percent (Supplementary Table 4). Overall, mechanical properties are excellent in some cases (Ag, Pd, $\mathrm{Pt})^{15}$, good in others ( $\mathrm{Al}, \mathrm{Au}, \mathrm{Ca}, \mathrm{Cu}, \mathrm{Pb}, \mathrm{Sr}$ ), and otherwise benefit from refinements. The reliability of the neat $12-6 \mathrm{LJ}$ model is overall good for mechanical properties (in the 9-6 $\mathrm{L}$ model, acceptable), considering the simplicity, computational efficiency, and compatibility with inorganic and biomolecular force fields.

Experimental reference data have typical uncertainties of $\pm 5 \%$, which can also be higher at $10-20 \%$ for some metals (Table 5 and Fig. 3). For Ac and Es, reference data were only partially or not available. These uncertainties in elastic constants $C_{11}, C_{12}, C_{44}$, and elastic moduli $E, K, G, v$ from experiments are clearly higher than for lattice parameters and surface energies ${ }^{59,81,82}$. The origins include: (1) Elastic properties are a function of stress and strain and usually defined in the limit of zero stress. Experimental techniques (acoustic, volume compression, etc) can involve non-negligible applied stresses and strains, similar to notable applied strains in 


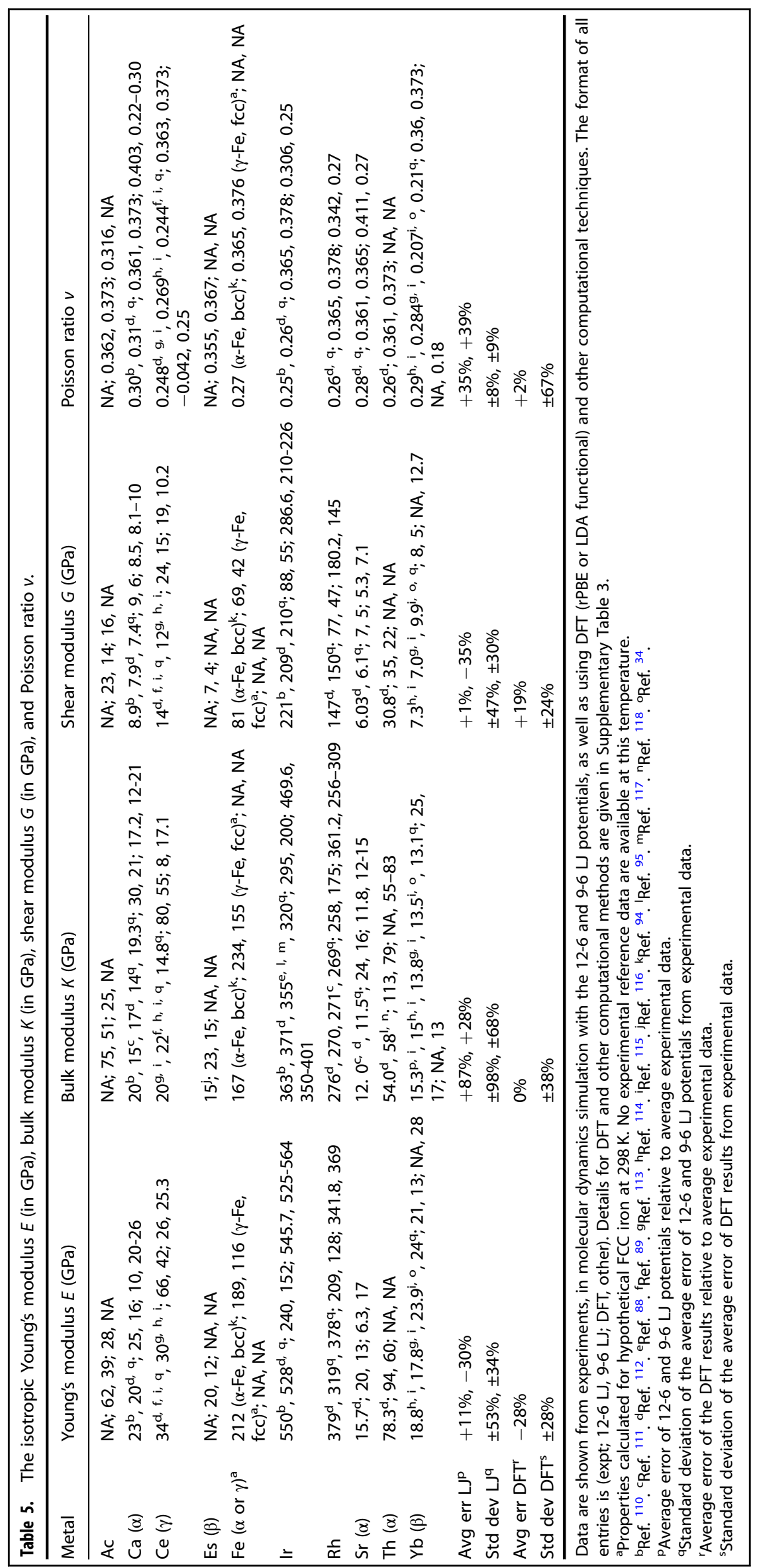


simulations (0.5-1\%). (2) The microstructure of the metal crystals and specimens in experiments can have imperfections and affect the results. (3) Young's modulus $E$ can have a strong dependence on the [hkl] direction due to the discrete atomic structure (Supplementary Note 5$)^{83}$. In addition, some random measurement error occurs and these differences explain some deviations. Fully quantitative comparisons of experiments with simulations would benefit from complete stress-strain curves and monitoring all elastic constants (focusing on $C_{11}, C_{12}$, and $C_{44}$ ) ${ }^{84,85}$.

Outstanding fits to mechanical properties have been obtained with recent EAMs that have uncertainties of $\sim 5 \%$ in $E, K, G$, and $v^{34}$. These EAMs, however, use 36 fit parameters per metal compared to 2 interpretable parameters in the LJ model. Mechanical properties computed using DFT and other computational techniques scatter by a comparable amount as the $L$ potentials (Table 5 and Supplementary Tables 3 and 4). Some deviations are under $5 \%$ and some in excess of $100 \%$ from the experiment, including surprising results such as a negative Poisson ratio for $\mathrm{Ce}$ (Table 5 and Supplementary Table 3$)^{86}$. We re-computed mechanical data for all $18 \mathrm{fcc}$ metals with the RPBE functional, which is not feasible for some heavier elements, and utilized the LDA functional for Ce (Table 5 and Supplementary Tables 3 and 5). DFT data have notable deviations from experiments and perform reasonably well with average errors of $-28 \pm 28 \%$ in Young's modulus, $0 \pm 38 \%$ in bulk modulus, $+19 \pm 24 \%$ in shear modulus, and $+2 \pm 67 \%$ in Poisson ratio relative to experiment.

Deviations of the LJ model in mechanical properties are related to simplicity. The level of sophistication is sufficient to simulate crystal structures, surfaces, interfaces with electrolytes and organic molecules, and interfacial properties of fcc alloys ${ }^{31}$ in excellent accuracy, unmatched by common DFT calculations and $10^{8}$ faster. The validation of crystal structures and surface energies supports reasonable estimates for elastic constants, which are the second derivatives of the energy with respect to coordinates. However, the reproduction of metal-specific Poisson ratios and anisotropies of mechanical properties requires the inclusion of more detail of the electronic structure. For example, the addition of a harmonically bonded virtual electron to gold atoms previously improved computed mechanical properties $5-10 \%$ closer to experimental values, in addition to accounting for image potentials and external electric fields on the fly (Supplementary Fig. $3 \mathrm{~m})^{16}$. The addition of four negatively charged virtual $d$ electrons in tetrahedral geometry around a positively charged $\mathrm{W}$ nucleus reproduces the structure of the bcc lattice as well as the specific Poisson ratio and elastic moduli (Supplementary Fig. 1I) ${ }^{50}$. Similar order-of-magnitude improvements are feasible through the representation of virtual $n$ electrons in water (TIP5P) ${ }^{87}$, organic compounds (PEO) ${ }^{41}$, and virtual $\pi$ electrons in graphite and aromatic molecules ${ }^{65}$. Going forward, a refined representation of metal atoms by combinations of a positively charged nucleus surrounded by specifically positioned, harmonically bonded negative point charges can yield more quantitative mechanical properties, add polarization, and preserve compatibility. Coverage of all $18 \mathrm{fcc}$ metals in the periodic table with simple $L J$ parameters and their evaluation is a first step towards this goal ${ }^{15}$.

\section{Iron and steel}

Iron occurs as a-Fe with a bcc structure at room temperature ${ }^{59}$. A stable fcc phase, $\gamma$-Fe (austenite), is formed in a temperature range from 912 to $1394^{\circ} \mathrm{C}$ (Supplementary Fig. 5) and above the eutectic point of $727^{\circ} \mathrm{C}$ in plain carbon steels ( $0.8 \mathrm{wt} \% \mathrm{C}$ ). The $\mathrm{LJ}$ models are the first validated atomistic models to simulate iron and its interfaces with electrolytes, oxides, and organic compounds (Table 1). They can also be used to simulate interfaces of bcc iron and fcc iron alloys with other metals using pairwise charge increments ${ }^{66}$. For bcc structures, we recommend fixing the positions of the metal atoms during molecular dynamics simulations due to voluntary conversion into an fcc structure, which is the energetically preferred structure for $L$ potentials. In fcc alloys, all atoms can be mobile as usual. Computed lattice parameters and surface energies for bcc Fe and fcc Fe show excellent agreement with experimental data as a function of temperature (Supplementary Fig. 5 and Supplementary Table 2).

Many structural applications of Fe involve austenitic stainless steels, which have an fcc structure $(\gamma-\mathrm{Fe})$ at room temperature, stabilized by the addition of small amounts of $\mathrm{Ni}, \mathrm{Mn}$, and $\mathrm{N}$. Austenitic steels account for most of the stainless steel production. For example, type 304 stainless steel with $18 \% \mathrm{Cr}$ and $8 \% \mathrm{Ni}$, as well as others containing $\mathrm{Mn}, \mathrm{N}, \mathrm{Mo}$, and $\mathrm{Nb}$, have optimized resistance to corrosion, pitting, and acid attack. To illustrate the application of the LJ parameters for iron, we prepared models of type 304 stainless steel with $18 \% \mathrm{Cr}$ and $8 \% \mathrm{Ni}$ content using the 12-6 LJ parameters and computed several properties (Fig. 4a-f). We also derived and validated new $\mathrm{LJ}$ parameters for $\mathrm{Cr}$, which can be applied to bcc $\mathrm{Cr}$ with fixed atoms and to fcc $\mathrm{Cr}$ in alloys with full atom mobility (Supplementary Table 6). The computed equilibrium density of type 304 stainless steel of $8.0 \mathrm{~g} \mathrm{~cm}^{-3}$ matches experimental data (Fig. $4 a)^{88}$ and the computed (111) surface energy of $2.29 \mathrm{~J} \mathrm{~m}^{-2}$ is close to experiments which suggest

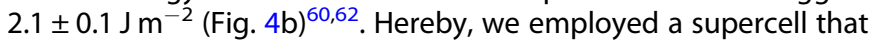
displays (111) surfaces along the z-axis. Furthermore, an aqueous interface was examined by the addition of 600 water molecules, which is present before superficial oxide formation (Fig. 4c). The steel-water interfacial tension of $\sim 1.8 \mathrm{~J} \mathrm{~m}^{-2}$ agrees with expectations from the experiment $\left(<2.0 \mathrm{~J} \mathrm{~m}^{-2}\right)$ and is close to the interface tension of pure Fe (Table 4). The bulk modulus is somewhat overestimated (Fig. 4a) and similar to that of pure iron (Fig. 3b) ${ }^{89}$. For a chemically realistic representation of the alloy, we used pairwise charge increments between neighbor atoms (Fig. 4d) (1) $^{31}$ that account for charge transfer between $\mathrm{Fe}, \mathrm{Cr}$, and $\mathrm{Ni}$ due to small differences in electronegativity ${ }^{31,90}$. Thereby, every atom is surrounded by 12 nearest neighbors in the bulk fcc-type crystal, while at the (111) surface the coordination number is reduced to 9 and leads to slightly different atomic charges (Fig. 4e, f). The impact of the atomic charges on the computed bulk properties of this particular alloy $(\mathrm{Fe}-\mathrm{Cr}-\mathrm{Ni})$ can be neglected in the first approximation as the electronegativities are similar. However, the differences are likely significant enough to affect the binding of corrosion inhibitors and the kinetics of surface reactions as even small differences in activation barriers $\Delta E_{\mathrm{a}}$ lead to large differences in reaction kinetics. For example, a difference of only $\Delta E_{\mathrm{a}}=+1 R T$ $\left(+0.6 \mathrm{kcal} \mathrm{mol}^{-1}\right)$ can delay corrosion by 2.7 times $\left(\sim e^{-\Delta E a / R T}\right)$. Further details are described in Supplementary Notes 6 and 7.

\section{Simulation of chemical reactions}

The earlier parameters for fcc metals have been used to model chemical reactions and covalent bonding to metal surfaces (Fig. $4 \mathrm{~g}-\mathrm{k})$. Molecular dynamics simulations can be locally coupled via QM/MM approaches to study bond breaking and transition states on metal surfaces (Fig. $4 \mathrm{~g})^{80}$. The region around the reaction center (darker color) was treated quantum mechanically, shown for the catalytic conversion of furfuryl alcohol to methylfurane. Covalent bonding of thiols (-S-R) to metal surfaces and nanoparticles can be represented by increasing the $\varepsilon_{0}$ values of sulfur to 0.5 or $1.0 \mathrm{kcal} \mathrm{mol}^{-1}$ using standard combination rules while all other bonded and nonbonded parameters of the thiol remain the same $(\sigma \sim 4 \AA$ for $S)$ (Fig. $4 \mathrm{~h}, \mathrm{i})^{91}$. Sulfur then preferentially coordinates epitaxial sites with a bond strength proportional to the surface energy of the metal, e. g., 10-16 kcal $\mathrm{mol}^{-1}$ on Pd (111). Specific reactions can also be studied on a case-by-case basis using knowledge about the reaction mechanism from experiments and ab-initio simulations. For example, the catalytic activity of a series of differently shaped palladium nanoparticles in Stille coupling reactions was predicted without 
Stainless Steel 304 Alloy $\left(\mathrm{Fe}_{366} \mathrm{Cr}_{98} \mathrm{Ni}_{40}\right)$

a

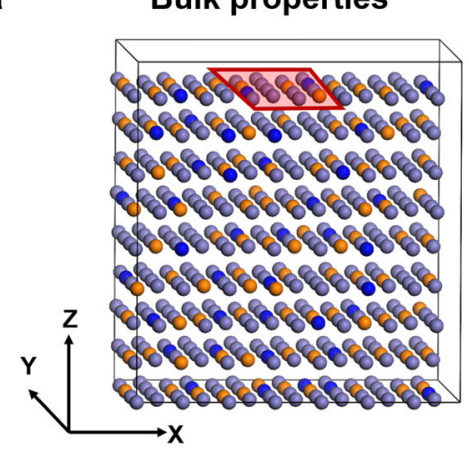

Density: $\quad 8.029 \mathrm{~g} \mathrm{~cm}^{-3}$ (sim.) 7.9-8.1 $\mathrm{g} \mathrm{cm}^{-3}$ (expt.) Bulk Modulus: $226 \mathrm{GPa}$ (sim.) $158 \mathrm{GPa}$ (expt)

\section{b Surface properties}

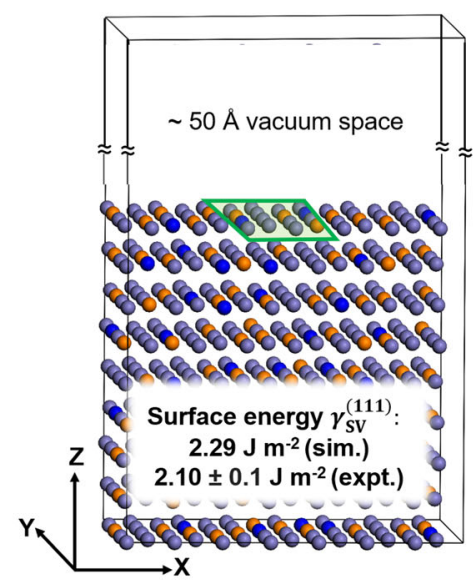

C Water interface

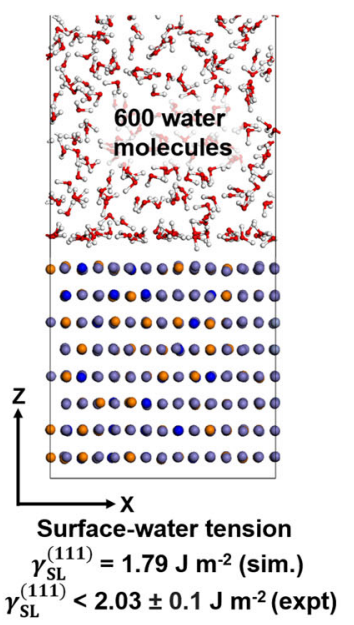

d

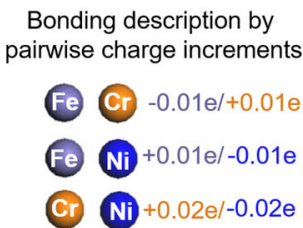

g

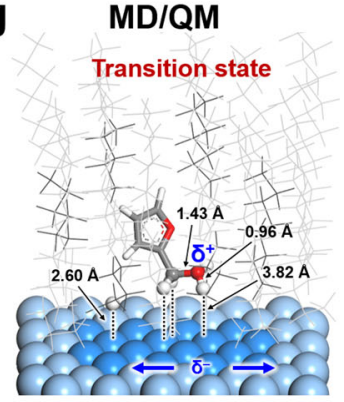

j Pd-catalyzed C-C coupling

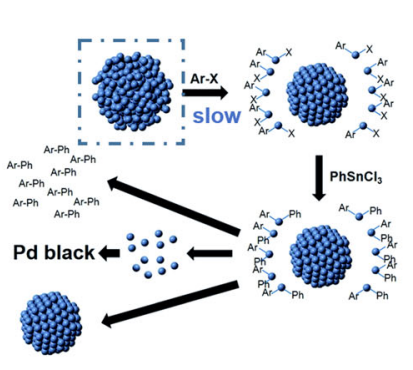

e

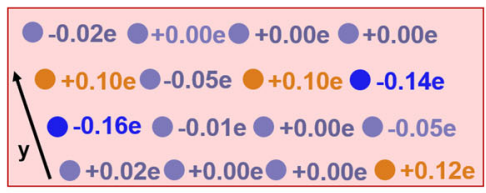

f

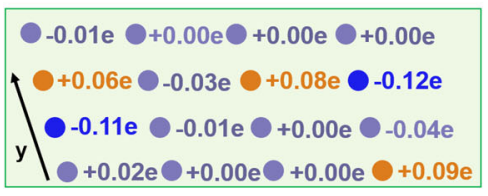

Examples of Reaction Modeling

h

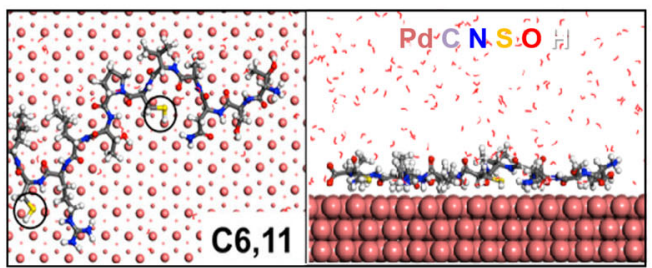

i

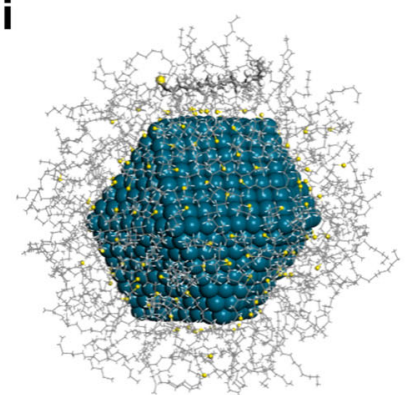

k

Oxidation \& corrosion
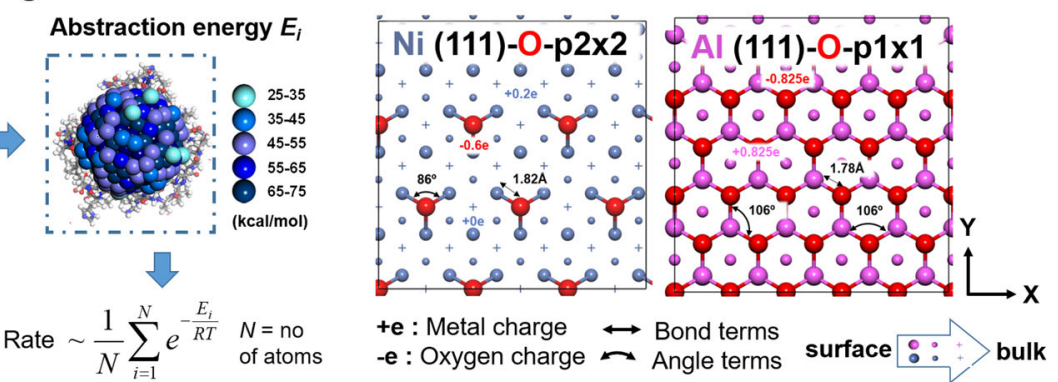

Fig. 4 Application of the $\mathrm{LJ}$ parameters for the simulation of stainless steel and chemical reactions. a-c Models of type 304 stainless steel (fcc Fe-Cr-Ni with $18 \mathrm{wt} \% \mathrm{Cr}$ and $8 \mathrm{wt} \% \mathrm{Ni}$ ) and computed bulk properties. Density ${ }^{88}$, surface energy ${ }^{60,62}$, and liquid interfacial properties agree very well with experimental data. The bulk modulus ${ }^{89}$ is overestimated (using 12-6 LJ parameters). $\mathbf{d}$-f Charge increments represent charge transfer between nearest-neighbor atoms ${ }^{31}$. Atomic charges are a function of the coordination number and differ for bulk atoms (highlighted atoms in $\mathbf{a}$ are shown in $\mathbf{e}$ ) versus surface atoms (highlighted atoms in $\mathbf{b}$ are shown in $\mathbf{f}$ ). The magnitude of pairwise charge increments depends on differences in atomization energy, ionization energy, and electron affinity. $\mathbf{g}$ MD/QM combinations to analyze local reactions ${ }^{80}$. The QM region is shown in a darker color. $\mathbf{h}, \mathbf{i}$ Covalently bound thiols to metal surfaces and nanoparticles can be represented by an increased $\mathrm{LJ}$ well depth for the sulfur atoms ${ }^{91}$. $\mathbf{j}$ Prediction of reaction rates of differently shaped Pd nanoparticles in C-C Stille coupling using knowledge about the rate-determining step and classical simulation ${ }^{24,25}$. $\mathbf{k}$ Representation of chemisorbed oxygen on aluminum and nickel surfaces using added bonded parameters based on experimental data ${ }^{92-95}$. 
the need for quantum-mechanical simulations (Fig. 4j) ${ }^{24,25}$. A detachment of $\mathrm{Pd}$ atoms from the nanoparticle was the ratelimiting (slowest) step and the abstraction energy could be directly calculated using the $L J$ potential. The exponential average over the surface atoms in the nanoparticle correlates well with the catalytic activity observed in the experiment (Fig. 4j). Oxidation and corrosion mechanisms can be described using models of partially oxidized surfaces with additional bonded terms, informed by structures from experiments and IFF parameter estimates (Fig. 4k) ) $^{92-95}$.

\section{DISCUSSION}

We introduced 12-6 and 9-6 Lennard-Jones parameters for the simulation of $10 \mathrm{fcc}$ metals including iron and the interfaces with electrolytes, organic, and inorganic compounds. Densities, surface energies, and interface energies are obtained in excellent agreement with the experiment under ambient conditions, with average deviations of $0.1 \%, 3 \%,<10 \%$, and mechanical properties in qualitative agreement. The parameters are defined at room temperature and atmospheric pressure, perform best within $298 \pm$ $200 \mathrm{~K}$, and can be modified for different conditions. The LJ parameters for fcc metals can be integrated into existing biomolecular and materials-oriented force fields such as IFF, AMBER, CHARMM, COMPASS, CVFF, DREIDING, OPLS-AA, and PCFF. Compatibility using standard combination rules has been extensively documented and no additional parameters are needed. We describe some applications, including the structures and energies of metal-water interfaces, metal-organic interfaces, the simulation of stainless steel alloys, and chemical reactions.

The Lennard-Jones models for fcc metals perform up to ten times more accurately than DFT and EAM models for surface and interfacial properties, and the computational efficiency is orders of magnitude higher due to using only 2 parameters per metal. High accuracy and interpretability originate from the relation of $\sigma$ and $\varepsilon_{0}$ to the density and surface energy of each metal. The models perform very well in the computation of quantities not included in the calibration, for example, the anisotropy of (111) and (100) surface energies, interfacial properties with electrolytes and organic molecules, and properties of alloys. Limitations include mechanical properties, which reach a variable agreement with experimental data. The agreement is good relative to experiment for metals with Poisson ratios close to the default Poisson ratio of LJ models (0.36-0.37), and 12-6 LJ parameters tend to perform better than 9-6 LJ parameters. Uncertainties in mechanical properties are comparable to DFT methods, and embedded atom models with 36 fit parameters per metal perform better ${ }^{34}$. The $\mathrm{LJ}$ models are the first models for atomistic simulations of some metals (Ac, Es, Th).

The simplicity of the models allows easy implementation in molecular dynamics and Monte Carlo simulations. The models are extensible to alloys using charge increments ${ }^{31}$, to covalent surface modification via thiols 24,91 , and chemical reactions ${ }^{24,25,96}$. This study of $10 \mathrm{fcc}$ metals, together with the earlier work for $8 \mathrm{fcc}$ metals ${ }^{15}$, covers all $18 \mathrm{fcc}$ elemental metals in the periodic table. The parameters also apply to fcc iron alloys such as stainless steels with full atom mobility, as well as to bcc iron and bcc chromium with fixed atoms. The method of parameterization is suitable for $\mathrm{fcc}$ phases of other metals at relevant temperatures and pressures. Going forward, extensions of the $\mathrm{L}$ models with bonded virtual electrons can cover a broader range of crystal structures (bcc and hcp metals), predict more accurate mechanical properties and on the fly polarization with a small increase in computational cost and no loss of existing attributes ${ }^{16,50}$.

While the LJ potentials do not explicitly incorporate the electronic structure, they implicitly incorporate some essential features of the electronic structure better than current DFT methods, such as correct surface energies. The simulation of chemical reactions at interfaces using the $L J$ parameters is possible on a case-by-case basis using key information about reaction steps from experiment and theory, without resorting to QM simulations $24,25,96,97$. Simulations of the full electronic structure during bond formation and bond breaking can be achieved using QM/MM approaches, or a combination of separate quantum-mechanical calculations at a local scale with snapshots taken from molecular dynamics simulations (QM/MD) ${ }^{80}$.

\section{METHODS}

\section{Model building}

Model systems of the metals of $1.5-3.0 \mathrm{~nm}$ side length were built from multiple unit cells, assuming crystal structure data obtained by X-ray diffraction ${ }^{59}$. Larger models were utilized to compute surface energies and interfaces with water. The Materials Studio program was used for model building and visualization.

\section{Simulation protocols}

We employed a spherical cutoff at $1.2 \mathrm{~nm}$ for the pairwise summation of $\square$ interactions. Lattice parameters were computed using the supercell and molecular dynamics simulation in the NPT ensemble at $298.15 \mathrm{~K}$ and $101.3 \mathrm{kPa}$ (Fig. 1a). Surface energies were computed using a set of two models, bulk models and cleaved models to create a surface, using molecular dynamics simulation in the NVT ensemble at $298.15 \mathrm{~K}$ and $101.3 \mathrm{kPa}$ (Fig. 1b). Solid-water interfacial energies were computed from the difference in average energies for 3D periodic boxes containing a metal-water interface, the bulk metal, and bulk water by molecular dynamics simulation in the NPT ensemble (Fig. 1c). Mechanical properties were computed using the Discover program and the LAMMPS program, which yield the same results within a typical difference of $0-3 \%$ (Fig. 1a). The simulation settings in Discover involved a time step of $1 \mathrm{fs}$, the summation of pairwise interactions with a spherical cutoff at $1.2 \mathrm{~nm}$, the summation of electrostatic interactions with Ewald summation in high accuracy $\left(10^{-6}\right)$, temperature control by velocity scaling with a temperature window of $10 \mathrm{~K}$, and the Parrinello-Rahman barostat in case of the NPT ensemble. Simulation settings in LAMMPS were equivalent, except for the PPPM method for electrostatic interactions $\left(10^{-6}\right.$ accuracy), Nose-Hoover chains for temperature control, and Nose-Hoover chains for pressure control in case of the NPT ensemble. Details of computational methods including DFT calculations of mechanical properties and a discussion of entropy contributions to surface and interfacial free energies are provided in the Supplementary Methods. Atomistic models, force field files, and input files to reproduce all data are available in the Supplementary Dataset.

\section{DATA AVAILABILITY}

All data generated or analyzed during this study are included in this published article, the Supplementary Information, and the Supplementary Dataset. Additional information can be requested from the corresponding author.

\section{CODE AVAILABILITY}

All codes utilized in this study are commercially available (Discover, CASTEP) and open source (LAMMPS). Run scripts and analysis scripts are provided in the Supplementary Dataset. Additional information can be requested from the corresponding author.

Received: 26 March 2020; Accepted: 12 December 2020; Published online: 29 January 2021

\section{REFERENCES}

1. Dreaden, E. C., Alkilany, A. M., Huang, X. H., Murphy, C. J. \& El-Sayed, M. A. The golden age: gold nanoparticles for biomedicine. Chem. Soc. Rev. 41, 2740-2779 (2012).

2. Huang, X. Q. et al. High-performance transition metal-doped Pt3Ni octahedra for oxygen reduction reaction. Science 348, 1230-1234 (2015). 
3. Li, M. F. et al. Ultrafine jagged platinum nanowires enable ultrahigh mass activity for the oxygen reduction reaction. Science 354, 1414-1419 (2016).

4. Heinz, H. et al. Nanoparticle decoration with surfactants: molecular interactions, assembly, and applications. Surf. Sci. Rep. 72, 1-58 (2017).

5. Murphy, C. J. et al. Anisotropic metal nanoparticles: synthesis, assembly, and optical applications. J. Phys. Chem. B 109, 13857-13870 (2005).

6. Auyeung, E. et al. DNA-mediated nanoparticle crystallization into Wulff polyhedra. Nature 505, 73-77 (2014).

7. Macfarlane, R. J., Jones, M. R., Lee, B., Auyeung, E. \& Mirkin, C. A. Topotactic interconversion of nanoparticle superlattices. Science 341, 1222-1225 (2013).

8. Zhu, E. et al. Long-range hierarchical nanocrystal assembly driven by molecular structural transformation. J. Am. Chem. Soc. 141, 1498-1505 (2019).

9. Zhu, E. et al. Peptide-assisted 2-D assembly toward free-floating ultrathin platinum nanoplates as effective electrocatalysts. Nano Lett. 19, 3730-3736 (2019).

10. Cao, L. et al. Differential surface elemental distribution leads to significantly enhanced stability of PtNi-based ORR catalysts. Matter 1, 1567-1580 (2019).

11. Sarikaya, M., Tamerler, C., Jen, A. K. Y., Schulten, K. \& Baneux, F. Molecular biomimetics: nanotechnology through biology. Nat. Mater. 2, 577-585 (2003).

12. Naik, R. R. et al. Peptide templates for nanoparticle synthesis derived from polymerase chain reaction-driven phage display. Adv. Funct. Mater. 14, 25-30 (2004).

13. Xia, Y., Xiong, Y., Lim, B. \& Skrabalak, S. E. Shape-controlled synthesis of metal nanocrystals: simple chemistry meets complex physics? Angew. Chem. Int. Ed. 48, 60-103 (2009)

14. Dacheux, N., Clavier, N. \& Podor, R. Monazite as a promising long-term radioactive waste matrix: benefits of high-structural flexibility and chemical durability. Am. Mineral. 98, 833-847 (2013).

15. Heinz, H., Vaia, R. A., Farmer, B. L. \& Naik, R. R. Accurate simulation of surfaces and interfaces of face-centered cubic metals using $12-6$ and $9-6$ LennardJones potentials. J. Phys. Chem. C. 112, 17281-17290 (2008).

16. Geada, I. L., Ramezani-Dakhel, H., Jamil, T., Sulpizi, M. \& Heinz, H. Insight into induced charges at metal surfaces and biointerfaces using a polarizable Lennard-Jones potential. Nat. Commun. 9, 716 (2018).

17. Feng, J. et al. Influence of the shape of nanostructured metal surfaces on adsorption of single peptide molecules in aqueous solution. Small 8, 1049-1059 (2012).

18. Feng, J. et al. Adsorption mechanism of single amino acid and surfactant molecules to $\mathrm{Au}\{111\}$ surfaces in aqueous solution: design rules for metalbinding molecules. Soft Matter 7, 2113-2120 (2011).

19. Heinz, H. et al. Nature of molecular interactions of peptides with gold, palladium, and Pd-Au bimetal surfaces in aqueous solution. J. Am. Chem. Soc. 131 9704-9714 (2009).

20. Roussel, T. J., Barrena, E., Ocal, C. \& Faraudo, J. Predicting supramolecular selfassembly on reconstructed metal surfaces. Nanoscale 6, 7991-8001 (2014).

21. Ramezani-Dakhel, H., Ruan, L. Y., Huang, Y. \& Heinz, H. Molecular mechanism of specific recognition of cubic Pt nanocrystals by peptides and the concentrationdependent formation from seed crystals. Adv. Funct. Mater. 25, 1374-1384 (2015).

22. Ruan, L. et al. Tailoring molecular specificity toward a crystal facet: a lesson from biorecognition toward Pt\{111\}. Nano Lett. 13, 840-846 (2013).

23. Chew, A. K. \& Van Lehn, R. C. Effect of core morphology on the structural asymmetry of alkanethiol monolayer-protected gold nanoparticles. J. Phys. Chem. C. 122, 26288-26297 (2018).

24. Bedford, N. M. et al. Elucidation of peptide-directed palladium surface structure for biologically tunable nanocatalysts. ACS Nano 9, 5082-5092 (2015).

25. Ramezani-Dakhel, H., Mirau, P. A., Naik, R. R., Knecht, M. R. \& Heinz, H. Stability, surface features, and atom leaching of palladium nanoparticles: toward prediction of catalytic functionality. Phys. Chem. Chem. Phys. 15, 5488-5492 (2013).

26. Zhang, C. et al. Low-temperature charging dynamics of the ionic liquid and its gating effect on FeSe0.5Te0.5 superconducting films. ACS Appl. Mater. Interfaces 11, 17979-17986 (2019).

27. Shi, W., Zhang, Z. \& Li, S. Quantitative prediction of position and orientation for platonic nanoparticles at liquid/liquid interfaces. J. Phys. Chem. Lett. 9, 373-382 (2018).

28. Meng, C., Liao, L. \& Huang, C. Study on failure mechanism of Cu-polyethylene-Cu sandwich structure by molecular dynamics simulation. Comput. Mater. Sci. 154, 315-324 (2018).

29. Zhu, Z. et al. Reversible hydrophobicity-hydrophilicity transition modulated by surface curvature. J. Phys. Chem. Lett. 9, 2346-2352 (2018).

30. Nathanson, M., Kanhaiya, K., Pryor, A., Miao, J. \& Heinz, H. Atomic-scale structure and stress release mechanism in core-shell nanoparticles. ACS Nano 12, 12296-12304 (2018)

31. Liu, J. et al. Understanding chemical bonding in alloys and the representation in atomistic simulations. J. Phys. Chem. C. 122, 14996-15009 (2018).
32. $\mathrm{Xu}, \mathrm{R}$. et al. Three-dimensional coordinates of individual atoms in materials revealed by electron tomography. Nat. Mater. 14, 1099-1103 (2015).

33. Shi, P. et al. Enhanced strength-ductility synergy in ultrafine-grained eutectic high-entropy alloys by inheriting microstructural lamellae. Nat. Commun. 10, 489 (2019).

34. Santodonato, L. J. et al. Deviation from high-entropy configurations in the atomic distributions of a multi-principal-element alloy. Nat. Commun. 6, 5964 (2015).

35. Sheng, H. W., Kramer, M. J., Cadien, A., Fujita, T. \& Chen, M. W. Highly optimized embedded-atom-method potentials for fourteen FCC metals. Phys. Rev. B 83, 134118 (2011).

36. Singh-Miller, N. E. \& Marzari, N. Surface energies, work functions, and surface relaxations of low-index metallic surfaces from first principles. Phys. Rev. B 80, 235407 (2009).

37. Silvestrelli, P. L. \& Ambrosetti, A. Van Der Waals corrected density functional theory simulation of adsorption processes on noble-metal surfaces: Xe on Ag (111), Au(111), and Cu(111). J. Low. Temp. Phys. 185, 183-197 (2016).

38. Ruiz, V. G., Liu, W. \& Tkatchenko, A. Density-functional theory with screened van Der Waals interactions applied to atomic and molecular adsorbates on closepacked and non-close-packed surfaces. Phys. Rev. B 93, 035118 (2016).

39. Liu, W., Tkatchenko, A. \& Scheffler, M. Modeling adsorption and reactions of organic molecules at metal surfaces. Acc. Chem. Res. 47, 3369-3377 (2014).

40. Grimme, S., Antony, J., Ehrlich, S. \& Krieg, H. A consistent and accurate ab initio parametrization of density functional dispersion correction (DFT-D) for the 94 elements H-Pu. J. Chem. Phys. 132, 154104 (2010).

41. Goerigk, L. \& Grimme, S. A thorough benchmark of density functional methods for general main group thermochemistry, kinetics, and noncovalent interactions. Phys. Chem. Chem. Phys. 13, 6670-6688 (2011).

42. Heinz, H., Lin, T.-J., Mishra, R. K. \& Emami, F. S. Thermodynamically consistent force fields for the assembly of inorganic, organic, and biological nanostructures: the interface force field. Langmuir 29, 1754-1765 (2013).

43. Lindorff-Larsen, K., Piana, S., Dror, R. O. \& Shaw, D. E. How fast-folding proteins fold. Science 334, 517-520 (2011).

44. Dauber-Osguthorpe, P. et al. Structure and energetics of ligand binding to proteins: Escherichia coli dihydrofolate reductase-trimethoprim, a drug-receptor system. Proteins Struct. Funct. Genet. 4, 31-47 (1988).

45. Sun, H., Mumby, S. J., Maple, J. R. \& Hagler, A. T. An ab-initio CFF93 all-atom force field for polycarbonates. J. Am. Chem. Soc. 116, 2978-2987 (1994).

46. Huang, J. \& MacKerell, A. D. CHARMM36 all-atom additive protein force field: validation based on comparison to NMR data. J. Comput. Chem. 34, 2135-2145 (2013).

47. Jorgensen, W. L., Maxwell, D. S. \& TiradoRives, J. Development and testing of the OPLS all-atom force field on conformational energetics and properties of organic liquids. J. Am. Chem. Soc. 118, 11225-11236 (1996).

48. Wang, J. M., Wolf, R. M., Caldwell, J. W., Kollman, P. A. \& Case, D. A. Development and testing of a general amber force field. J. Comput. Chem. 25, 1157-1174 (2004).

49. Mayo, S. L., Olafson, B. D. \& Goddard, W. A. DREIDING: a generic force field for molecular simulations. J. Phys. Chem. 94, 8897-8909 (1990).

50. Sun, H. COMPASS: an ab initio force-field optimized for condensed-phase applications - overview with details on alkane and benzene compounds. J. Phys. Chem. B 102, 7338-7364 (1998).

51. Foiles, S. M., Baskes, M. I. \& Daw, M. S. Embedded atom-method functions for the FCC metals Cu, Ag, Au, Ni, Pd, Pt, and their alloys. Phys. Rev. B 33, 7983-7991 (1986).

52. Daw, M. S., Foiles, S. M. \& Baskes, M. I. The embedded atom method: a review of theory and applications. Mater. Sci. Rep. 9, 251-310 (1993).

53. Heinz, H. \& Ramezani-Dakhel, H. Simulations of inorganic-bioorganic interfaces to discover new materials: insights, comparisons to experiment, challenges, and opportunities. Chem. Soc. Rev. 45, 412-448 (2016).

54. Ghiringhelli, L. M. \& Delle Site, L. Phenylalanine near inorganic surfaces: conformational statistics vs specific chemistry. J. Am. Chem. Soc. 130, 2634-2638 (2008).

55. Pensado, A. S. \& Padua, A. A. H. Solvation and stabilization of metallic nanoparticles in ionic liquids. Angew. Chem. Int. Ed. 50, 8683-8687 (2011).

56. Wright, L. B., Rodger, P. M., Corni, S. \& Walsh, T. R. GolP-CHARMM: first-Principles based force fields for the interaction of proteins with $\mathrm{Au}(111)$ and $\mathrm{Au}(100) . \mathrm{J}$. Chem. Theor. Comput. 9, 1616-1630 (2013).

57. Born, M. Eine Thermochemische Anwendung der Gittertheorie. Verh. Dtsch. Phys. Ges. 21, 13-24 (1919).

58. Hirschfelder, J. O., Curtiss, C. F. \& Bird, R. B. Molecular Theory of Gases and Liquids (John Wiley, 1954).

59. Lide, D. R. CRC Handbook of Chemistry and Physics, 96th edn. (CRC Press, 2015)

60. Tyson, W. R. \& Miller, W. A. Surface free-energies of solid metals - estimation from liquid surface-tension measurements. Surf. Sci. 62, 267-276 (1977). 
61. Keene, B. J. Review of data for the surface tension of pure metals. Int. Mater. Rev. 38, 157-192 (1993).

62. Mills, K. C. \& Su, Y. C. Review of surface tension data for metallic elements and alloys: part 1 - pure metals. Int. Mater. Rev. 51, 329-351 (2006).

63. Jha, K. C., Liu, H., Bockstaller, M. R. \& Heinz, H. Facet recognition and molecular ordering of ionic liquids on metal surfaces. J. Phys. Chem. C. 117, 25969-25981 (2013).

64. Emami, F. S. et al. Force field and a surface model database for silica to simulate interfacial properties in atomic resolution. Chem. Mater. 26, 2647-2658 (2014).

65. Pramanik, C., Gissinger, J. R., Kumar, S. \& Heinz, H. Carbon nanotube dispersion in solvents and polymer solutions: mechanisms, assembly, and preferences. ACS Nano 11, 12805-12816 (2017).

66. Chen, J. et al. Building two-dimensional materials one row at a time: avoiding the nucleation barrier. Science 362, 1135-1139 (2018).

67. Heinz, H., Koerner, H., Anderson, K. L., Vaia, R. A. \& Farmer, B. L. Force field for mica-type silicates and dynamics of octadecylammonium chains grafted to montmorillonite. Chem. Mater. 17, 5658-5669 (2005).

68. Lee, J. et al. CHARMM-GUI input generator for NAMD, GROMACS, AMBER, OpenMM, and CHARMM/OpenMM simulations using the CHARMM36 additive force field. J. Chem. Theory Comput. 12, 405-413 (2016).

69. Jo, S., Kim, T., Iyer, V. G. \& Im, W. CHARMM-GUI: a web-based graphical user interface for CHARMM. J. Comput. Chem. 29, 1859-1865 (2008).

70. Zhou, J. et al. Observing crystal nucleation in four dimensions using atomic electron tomography. Nature 570, 500-503 (2019).

71. Mehl, M. J. \& Papaconstantopoulos, D. A. Applications of a tight-binding totalenergy method for transition and noble metals: elastic constants, vacancies, and surfaces of monatomic metals. Phys. Rev. B 54, 4519 (1996).

72. Schneemilch, M. \& Quirke, N. Free energy of adsorption of supported lipid bilayers from molecular dynamics simulation. Chem. Phys. Lett. 664, 199-204 (2016).

73. Menon, S. K. \& Martin, P. L. Determination of the anisotropy of surface free energy of fine metal particles. Ultramicroscopy 20, 93-98 (1986).

74. Flueli, M. \& Borel, J.-P. Surface energy anisotropy measurements on a small cuboctahedron of gold observed by high resolution electron microscopy (HREM). J. Cryst. Growth 91, 67-70 (1988).

75. Lee, W. H. et al. The equilibrium shape and surface energy anisotropy of clean platinum. J. Catal. 126, 658-671 (1990).

76. Mezey, L. Z. \& Giber, J. The surface free-energies of solid chemical elements calculation from internal free enthalpies of atomization. Jpn. J. Appl. Phys. Part 1 21, 1569-1571 (1982).

77. Osman, M. A. \& Keller, B. A. Wettability of native silver surfaces. Appl. Surf. Sci. 99, 261-263 (1996).

78. Heinz, H., Jha, K. C., Luettmer-Strathmann, J., Farmer, B. L. \& Naik, R. R. Polarization at metal-biomolecular interfaces in solution. J. R. Soc. Interface 8, 220-232 (2011).

79. Dharmawardhana, C. C. et al. Reliable computational design of biologicalinorganic materials to the large nanometer scale using interface-FF. Mol. Sim. 43, 1394-1405 (2017).

80. Mark, L. O., Zhu, C., Medlin, J. W. \& Heinz, H. Understanding the surface reactivity of ligand-protected metal nanoparticles for biomass upgrading. ACS Catal. 10, 5462-5474 (2020).

81. Kosevitch, A. M., Lifshitz, E. M., Landau, L. D. \& Pitaevskii, L. P. Theory of Elasticity 3rd edn, Vol. 7 (Butterworth-Heinemann, 1999).

82. Simmons, G. \& Wang, H., eds. Single Crystal Elastic Constants and Calculated Aggregate Properties: A Handbook 2nd edn (MIT Press, 1971).

83. Hertzberg, R. W. Deformation and Fracture Mechanics of Engineering Materials, 3rd edn. (John Wiley \& Sons, 1989).

84. Mishra, R. K., Fernández-Carrasco, L., Flatt, R. J. \& Heinz, H. A force field for tricalcium aluminate to characterize surface properties, initial hydration, and organically modified interfaces in atomic resolution. Dalton Trans. 43, 10602-10616 (2014).

85. Liu, J. et al. Interpretable molecular models for molybdenum disulfide and insight into selective peptide recognition. Chem. Sci. 11, 8708-8722 (2020).

86. Blanca, E., Rodriguez, C. O., Shitu, J. \& Novikov, D. L. Degree of localization of the exchange-correlation hole and its influence on the ground-state (structural and magnetic) properties of d. Met. J. Phys. Condens. Mat. 13, 9463-9470 (2001)

87. Mahoney, M. W. \& Jorgensen, W. L. A five-site model for liquid water and the reproduction of the density anomaly by rigid, nonpolarizable potential functions. J. Chem. Phys. 112, 8910-8922 (2000).

88. O. Oyj. Handbook of Stainless Steel (Outokumpu Oyj, Espoo, Finland, 2013).

89. Ledbetter, H. \& Austin, M. Effects of carbon and nitrogen on the elastic constants of AISI type 304 stainless steel. Mat. Sci. Eng. 70, 143-149 (1985).

90. Heinz, H. \& Suter, U. W. Atomic charges for classical simulations of polar systems. J. Phys. Chem. B 108, 18341-18352 (2004)
91. Coppage, R. et al. Exploiting localized surface binding effects to enhance the catalytic reactivity of peptide-capped nanoparticles. J. Am. Chem. Soc. 135, 11048-11054 (2013).

92. Grimsby, D. V., Wu, Y. \& Mitchell, K. A LEED analysis for the Ni (111) $-(2 \times 2)-\mathrm{O}$ surface structure: evidence for oxygen-induced relaxations of both vertical and lateral types in the close packed surface layer of nickel. Surf. Sci. 232, 51-55 (1990).

93. Narusawa, T., Gibson, W. \& Törnqvist, E. Structure study of oxygen-adsorbed Ni (111) surface by high energy ion scattering. Surf. Sci. 114, 331-348 (1982).

94. Batra, I. P. \& Kleinman, L. Chemisorption of oxygen on aluminum surfaces. J. Electron Spectrosc. Relat. Phenom. 33, 175-241 (1984).

95. Martinson, C., Flodström, S., Rundgren, J. \& Westrin, P. Oxygen chemisorption on aluminum single crystals: site determination by LEED studies. Surf. Sci. 89, 102-113 (1979).

96. Ramezani-Dakhel, H. et al. Nature of peptide wrapping onto metal nanoparticle catalysts and driving forces for size control. Nanoscale 9, 8401-8409 (2017).

97. Briggs, B. et al. Atomic-scale identification of Pd leaching in nanoparticle catalyzed CC coupling: effects of particle surface disorder. Chem. Sci. 6, 6413-6419 (2015).

98. Basinski, Z. S., Hume-Rothery, W. \& Sutton, A. The lattice expansion of iron. $P$. Roy. Soc. A Math. Phy. 229, 459-467 (1955).

99. Skriver, H. L. \& Rosengaard, N. Surface energy and work function of elemental metals. Phys. Rev. B 46, 7157 (1992).

100. Aghemenlo, H., lyayi, S. \& Avwiri, G. Surface relaxation and surface energy of face-centered cubic. Met. J. Appl. Sci. Environ. Manag. 10, 37-42 (2006).

101. Karolewski, M. A. Tight-binding potentials for sputtering simulations with FCC and BCC metals. Radiat. Eff. Defects Solids 153, 239-255 (2001).

102. Needs, R. J., Godfrey, M. J. \& Mansfield, M. Theory of surface stress and surface reconstruction. Surf. Sci. 242, 215-221 (1991).

103. Baskes, M. I. Modified embedded-atom potentials for cubic materials and impurities. Phys. Rev. B 46, 2727-2742 (1992).

104. Methfessel, M., Hennig, D. \& Scheffler, M. Trends of the surface relaxations, surface energies, and work functions of the $4 \mathrm{~d}$ transition metals. Phys. Rev. B 46, 4816 (1992).

105. Polatoglou, H. M., Methfessel, M. \& Scheffler, M. Vacancy-formation energies at the (111) surface and in bulk Al, Cu, Ag, and Rh. Phys. Rev. B 48, 1877-1883 (1993).

106. Tran, R. et al. Surface Energies Of Elemental Crystals. Sci. Data 3, 160080 (2016).

107. Ropo, M., Kokko, K. \& Vitos, L. Assessing the Perdew-Burke-Ernzerhof Exchangecorrelation density functional revised for metallic bulk and surface systems. Phys. Rev. B 77, 195445 (2008).

108. Yoo, S.-H., Lee, J.-H., Jung, Y.-K. \& Soon, A. Exploring stereographic surface energy maps of cubic metals via an effective pair-potential approach. Phys. Rev. B 93, 035434 (2016).

109. Vitos, L., Ruban, A. V., Skriver, H. L. \& Kollár, J. The surface energy of metals. Surf. Sci. 411, 186-202 (1998).

110. Mehl, M. J., Klein, B. M. \& Papaconstantopoulos, D. A. First-principles calculation of elastic properties. Intermet. Compd. 1, 195-210 (1994).

111. Moruzzi, V., Janak, J. \& Schwarz, K. Calculated thermal properties of metals. Phys. Rev. B 37, 790 (1988).

112. Brandes, E. A. \& Brook, G. B. Smithells Metals Reference Book, 7th edn. (Butterworth Heinemann, 1992).

113. Gust, W. \& Royce, E. New electronic interactions in rare-earth metals at high pressure. Phys. Rev. B 8, 3595 (1973).

114. Eyring, L., Gschneidner, K. A. \& Lander, G. H. Handbook on the Physics and Chemistry of Rare Earths, Vol. 32 (Elsevier, 2002).

115. Haire, R. G. In The Chemistry of the Actinide and Transactinide Elements (eds Morss, L. R., Edelstein, N. M. \& Fuger, J.) Ch. 12, 1577-1620 (Springer, 2006).

116. Ledbetter, H. M. \& Reed, R. P. Elastic properties of metals and alloys, I. iron, nickel, and iron-nickel alloys. J. Phys. Chem. Ref. Data 2, 531-618 (1973).

117. Armstrong, P., Carlson, O. \& Smith, J. Elastic constants of thorium single crystals in the range $77-400^{\circ}$ K. J. Appl. Phys. 30, 36-41 (1959).

118. Stephens, D. The compression of some rare earth elements. J. Phys. Chem. Solids 25, 423-429 (1964)

\section{ACKNOWLEDGEMENTS}

We acknowledge support by the National Science Foundation (CBET 1530790, DMREF 1623947, OAC 1931587, CMMI 1940335) and the Office of Naval Research (ONR-MURIN00014-14-1-0675). This work utilized the Summit supercomputer, a joint effort of the University of Colorado Boulder and Colorado State University, which is supported by the National Science Foundation (ACl-1532235 and ACl-1532236). This work also used resources at the Argonne Leadership Computing Facility, which is a DOE Office of Science User Facility supported under Contract DE-AC02-06CH11357. 


\section{AUTHOR CONTRIBUTIONS}

H.H. conceived the study. K.K. and H.H. carried out simulations. S.K. and W.I. conceived and developed the Nanomaterial Modeler in CHARMM-GUI. K.K., W.I., and H.H. wrote the paper.

\section{COMPETING INTERESTS}

The authors declare no competing interests.

\section{ADDITIONAL INFORMATION}

Supplementary information The online version contains supplementary material available at https://doi.org/10.1038/s41524-020-00478-1.

Correspondence and requests for materials should be addressed to H.H.

Reprints and permission information is available at http://www.nature.com/ reprints
Publisher's note Springer Nature remains neutral with regard to jurisdictional claims in published maps and institutional affiliations.

Open Access This article is licensed under a Creative Commons Attribution 4.0 International License, which permits use, sharing, adaptation, distribution and reproduction in any medium or format, as long as you give appropriate credit to the original author(s) and the source, provide a link to the Creative Commons license, and indicate if changes were made. The images or other third party material in this article are included in the article's Creative Commons license, unless indicated otherwise in a credit line to the material. If material is not included in the article's Creative Commons license and your intended use is not permitted by statutory regulation or exceeds the permitted use, you will need to obtain permission directly from the copyright holder. To view a copy of this license, visit http://creativecommons. org/licenses/by/4.0/.

(c) The Author(s) 2021, corrected publication 2021 